\title{
Demand uncertainty in Robust Home Care Optimization
}

\author{
Paola Cappanera* \\ Dipartimento di Ingegneria dell'Informazione, Università di Firenze, \\ Via di Santa Marta 3, 50139 Firenze, Italy, \\ paola.cappanera@unifi.it
Maria Grazia Scutellà, Federico Nervi, Laura Galli
Dipartimento di Informatica, Università di Pisa,
Largo B. Pontecorvo 3, 56127 Pisa, Italy, scut@di.unipi.it,laura.galli@unipi.it \\ $\star$ Corresponding author
}

July 5, 2017

\begin{abstract}
We study the Home Care Problem under uncertainty. Home Care refers to medical, paramedical and social services that may be delivered to patient homes. The term includes several aspects involved in the planning of home care services, such as caregiver-to-patient assignment, scheduling of patient requests, and caregiver routing. In Home Care, cancellation of requests and additional demand for known or new patients are very frequent. Thus, managing demand uncertainty is of paramount importance in limiting service disruptions that might occur when such events realize. We address uncertainty of patient demand over a multiple-day time horizon, when assignment, scheduling and routing decisions are taken jointly, both from a methodological and a computational perspective. In fact, we propose a non-standard cardinality-constrained robust approach, analyse its properties, and report the results of a wide experimentation on real-life instances. The obtained results show that, for instances of moderate size, the approach is able to efficiently determine robust solutions of good quality in terms of balancing among caregivers and number of uncertain requests that can be managed. Also, the robustness of the solutions with respect to possible realizations of uncertain requests, evaluated on a small subset of instances, appears to be significant. Furthermore, preliminary experiments on a decomposition method, obtained from the robust one by fixing the scheduling decisions, show a drastic gain in computational efficiency, with the determination
\end{abstract}


of robust solutions of still good quality. Therefore, the approach appears to be very promising to cope with robustness even on Home Care instances of larger size.

Keywords: Home Care, Patient Demand Uncertainty, Robust Optimization

\section{Introduction}

The design of efficient Home Care Services is a relatively new and very challenging area of study in Operations Research, which is motivated by the ever increasing age of population and the consequent need to reduce hospitalization costs. The term Home Care Services refers to medical, paramedical and social services that may be delivered to patient homes. It comprises several aspects, such as caregiver-topatient assignment, scheduling of patient requests, and caregiver routing. Hence, the overall Home Care decisions are very complex to address in a rigorous and formal way. For this reason, the different planning aspects have generally been considered separately in the literature (e.g., [18]). Furthermore, whereas there now exists a fairly large number of heuristic procedures (see for example [2], [5], [11], [13], [14], [23], [24], [25], [27], [29]), very few exact approaches have been proposed dealing simultaneously with the different planning aspects in Home Care. We mention here [16], [28] (on a daily perspective) and [32], by referring to [15] for a recent and comprehensive review on Home Care planning.

An additional exact approach has been designed in [7], where the authors use the concept of pattern to jointly address: (i) the assignment of caregivers to patients; (ii) the scheduling of the patient requests in a given planning horizon; and (iii) the construction of the daily tours for the caregivers, indicating the sequence of patients that each caregiver must visit every day. Patterns have been used also as a decomposition tool to solve larger instances than the ones addressed in [7], leading to pattern-based two phase approaches to Home Care [34].

Although the Home Care problem literature is now reasonably broad, very few works address the issue of uncertainty in the problem data. Precisely, in [10] the problem of assigning a set of patients to a set of caregivers over a time horizon is considered, imposing that exactly one caregiver is assigned to each patient, and assuming that the amount of working time required by each patient is uncertain. A cardinality-constrained robust assignment model is proposed and tested on real-life instances. In [35], the Home Care scheduling and routing problem with stochastic service times is addressed. A stochastic programming model with recourse is proposed, which is then transformed into a classical set partitioning problem and an elementary shortest path problem with resource constraints, by approximating the expected penalty function for late arrival at a patient. A branch and price approach is designed and validated by means of numerical experiments. The case of stochastic service times is also addressed in [30], where the authors propose a 
two-stage approach, based on integer linear stochastic programming, to deal with staff dimensioning in Home Care. Finally, uncertainty on the availability of the caregivers is considered in [26], where a matheuristic is proposed to handle the case where caregivers are unexpectedly unable to operate. We conclude this short survey on uncertainty in Home Care by mentioning [31], where a robust optimization model for dispatching technicians under stochastic service times is proposed within a branch and price framework. In fact, the paper refers to a problem that, although related to a different application context, presents some similarities to the Home Care one.

The goal of this paper is to fill the literature gap between papers dealing with deterministic Home Care scenarios and papers addressing uncertainty in Home Care, by studying the Home Care problem where caregiver-to-patient assignment, scheduling of patient requests, and caregiver routing are taken into account jointly over a given multiple-day time horizon, and patient demand is subject to uncertainty. In particular, we generalize the cardinality-constrained robust framework proposed in [3] to extend the joint approach of [7] in an original manner. To the best of our knowledge this is the first time that a robust framework is applied to the Home Care problem, when assignment, scheduling and routing decisions are jointly addressed over a given time horizon. More generally, this is the first time that a cardinalityconstrained robust framework is applied, in the proposed form, to a complex vehicle routing problem such as the one arising in Home Care. We think that such a generalized robust framework could inspire the proposal of similar robust models for other relevant routing problems ([17],[6]).

We remark also that scenarios with uncertain requests have already been addressed in the Vehicle Routing literature, but with different characteristics with respect to the ones considered in this paper, and solely within the framework of stochastic optimization. For example, in [1] the authors assume that some customers are known at planning time, while others are dynamic. The goal is to service as many customers as possible given a fixed number of vehicles, assuming that stochastic information is available for the dynamic customers. A multiple scenario approach is proposed which continuously generates and solves scenarios that include both static and dynamic requests, and the plan is selected via a consensus function. On the other hand, [12] presents a two-stage recourse problem in which the vehicle routes are selected before the uncertain customer demands are realized. Once the demands are observed, a recourse decision is taken that amends the routes with returns to the depot, and a Tabu search heuristic is proposed to solve the problem. A similar recourse function is proposed in [21], which resembles the kind of robustness studied in this work, but for stochastic optimization problems: in case of failure, the recourse is to go to the depot and continue the route. Finally, for scenarios with mandatory as well as optional customers, and assuming stochastic travel and service 
times, the recently published paper [4] suggests to design initial routes serving the mandatory customers, and then to perform insertions of the optional customers between mandatory customers, with the intent of skipping some optional customers in case the realized service and travel times are larger than the planned ones. Skipping is based on a dynamic programming approach.

The structure of the paper is as follows. The deterministic or nominal Home Care problem studied in this paper is described in Section 2. ${ }^{1}$ Then, a robust Home Care counterpart is presented in Section 3 together with the robust approach we propose, which generalizes the cardinality-constrained one. The results of computational experiments on real-life instances are given in Section 4, and some concluding remarks are made in Section 5.

\section{Nominal Home Care}

The nominal Home Care Problem (HCP), as introduced in [7], is defined on a complete directed network $G=(N \cup\{0\}, A)$, where each node $j \in N=\{1, \ldots n\}$ corresponds to a patient. The node 0 is used to denote the "depot". All caregivers start their tour from the depot and come back to the depot at the end of their working day, for each day of the planning horizon $W$. Each caregiver is characterized by a "skill" representing the level of treatment the caregiver can provide when assisting a patient. A hierarchical structure of the set $K$ of skills is assumed, so that a caregiver with skill $k$ can work requests with skill up to $k$. In the following, $O$ will denote the set of the caregivers available in the planning horizon, and $O_{d} \subseteq O$ will be the subset of the caregivers available on day $d$, for each $d \in W$. Moreover, we are given a care plan $r_{j}$ for each patient $j \in N$. The care plan associated with patient $j$ specifies the number of visits of each skill required by $j$ in the planning horizon.

In [7], assignment, scheduling and routing decisions are "linked" by means of the concept of pattern, which represents a template for scheduling the patient requests along $W$. More precisely, it is assumed that, for each patient $j$, the requests expressed by the care plan $r_{j}$ can be operated according to a set $P_{j}$ of a priori given patterns. Formally, denoting by $P=\cup_{j=1}^{n} P_{j}$ the set of all patterns, and assuming that each patient requires at most one visit per day, each pattern $p \in P$ is such that $p(d)=0$ if no service is offered on day $d$, while $p(d)=k$ indicates that a visit of skill $k$ is operated according to pattern $p$ on day $d$. For example, if $r_{j}=(2,1)$, that is $j$ requires two visits of skill 1 and one visit of skill 2 , and if $W$ is a week, from Monday to Friday, then the requests of $j$ could be operated according to the pattern $(1,0,1,0,2)$, indicating that the two visits of skill 1 will be delivered on Monday and

\footnotetext{
${ }^{1}$ We emphasize that, although "deterministic" and "nominal" are synonym in this context, "nominal" will be preferably used, since this is the denomination commonly adopted in the robust optimization literature [3].
} 
on Wednesday, whereas the visit of skill 2 will be delivered on Friday. The pattern $p \in P_{j}$, selected for patient $j$, thus determines the scheduling of the requests of $j$, and it has to be compatible with his care plan, guaranteeing the required number of visits of appropriate skill.

Given the input data above, HCP thus consists of: $(i)$ assigning a pattern from $P_{j}$ to each patient $j$, so scheduling the requests of $j$, defined by the care plan $r_{j}$, during the planning horizon (care plan scheduling); (ii) assigning a caregiver to each patient $j$ for each day of the planning horizon in which a request of $j$ has been scheduled (caregiver assignment); (iii) determining the tour of each caregiver for each scheduled day (routing decisions).

In addressing assignment, scheduling and routing decisions, the skill constraints (i.e., the compatibility between the skills associated with the patient requests and the skills of the caregivers), the continuity of care (i.e., at most $T$ caregivers can be assigned to each patient in $W$, for a given $T$ ), and caregiver workday length constraints, taking into account the travel time along the links of the network, and the service time at the patients, must be satisfied. The objective function guiding the three groups of decisions is based on a social equity criterion, i.e. the caregiver workload balancing. Alternative objective functions, e.g. based on operating cost minimization, could be also considered [9].

Below we list the main HCP notation. The same notation will be used, in the next section, to define the proposed Home Care robust counterpart.

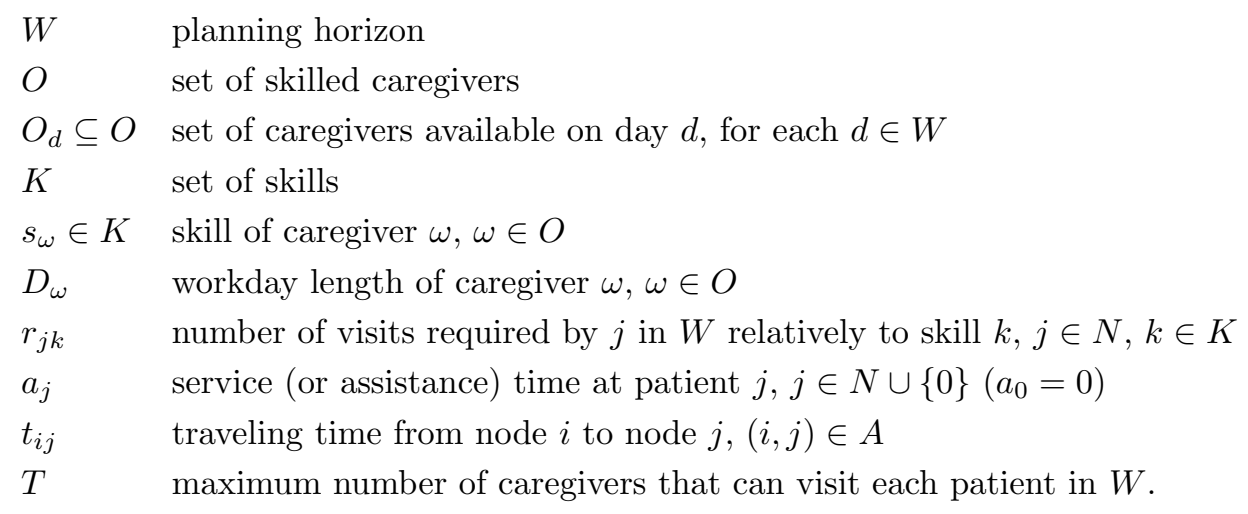

\section{Robust Home Care}

\subsection{Why robustness?}

In HCP, the input data are assumed to be certain. In particular, all patient care plans are assumed to be known in advance. However, in some practical settings, this assumption may be unrealistic. Indeed, patient requests are usually subject to uncertainty in the considered time horizon. Precisely, at the moment of planning, 
some requests are certain, meaning that we know for sure that they will need to be served, while others are uncertain. The uncertain requests can represent the arrival of additional demand for known or new patients. This additional demand is usually estimated, at the beginning of the planning horizon, by means of suitable forecasting tools [20]. However, uncertain requests can also model demand, for patients already assisted, that might be cancelled. This happens in contexts mainly dedicated to palliative care and terminal patients, due to the nature of the eligible patients.

In both cases, if we want to adopt a deterministic approach, we have two options: either disregard the uncertain requests and solely optimize on the certain ones, or consider all requests (including the uncertain ones) as certain. Clearly the first approach, that only serves certain requests, will produce cheaper solutions; yet, if any of the uncertain requests is confirmed later on during the time horizon, likely these may not be met, giving rise to service disruptions. The second approach, on the other hand, is very conservative, being able to serve all the uncertain requests, but producing very costly plans that may turn out to be strongly underused. Moreover, trying to plan all the uncertain requests can lead to unfeasibility, if the caregiver workday lengths are not sufficient to satisfy all of them.

Therefore, we look for a trade-off between solution cost and feasibility. This is the natural setting for Robust Optimization. The literature on Robust Optimization nowadays is vast and many different robust frameworks can be chosen. We decided to suitably generalize Bertsimas \& Sim framework [3], also known as $\Gamma$-robustness or cardinality-constrained robustness, since it offers good control on the degree of conservatism of the computed solutions. This will be deeply specified next.

\subsection{The sequence-preserving $\Gamma$-Robust Home Care Problem}

In order to take into account the uncertainty of some patient requests, we generalize the HCP, described in Section 2, as follows: each patient $j$ is now associated with both a certain care plan $\bar{r}_{j}$, corresponding to the certain skilled requests of $j$, and an uncertain care plan $\tilde{r}_{j}$, corresponding to the uncertain skilled requests of $j$. To model this, we duplicate each node of the logistic network, by associating each patient $j \in\{1, \ldots, n\}$ with a corresponding uncertain "copy", denoted by $j+n$. We therefore associate the "original" (i.e., certain) copy $j \in \bar{N}=\{1, \ldots, n\}$ with the certain skilled requests in $\bar{r}_{j}$, and the uncertain copy $j+n \in \tilde{N}=\{n+1, \ldots, 2 n\}$ with the uncertain skilled requests in $\tilde{r}_{j}$. Notice that $\tilde{r}_{j}$ or $\bar{r}_{j}$ could be missing for a patient $j$ : the first case verifies if $j$ is a deterministic patient, whereas the latter can be used to model $j$ as a new patient. A pattern is then assigned to each node in $\bar{N}$ as well as to each node in $\tilde{N}$, to schedule certain and uncertain requests, respectively.

Consider a set of tours, over the planning horizon, which includes all the certain and the uncertain requests, and a caregiver assignment to these tours. As we already observed, these tours represent a very conservative solution, since all the 
uncertain requests are scheduled. On the other hand, this is unlikely to happen, and only a subset of the uncertain requests will realize, making this kind of solution unnecessarily expensive.

To achieve a compromise between degree of robustness and solution cost, we consider scenarios where at most $\Gamma$ uncertain requests (or, equivalently, at most $\Gamma$ nodes from the set $\tilde{N}$ ) in each tour may realize, where $\Gamma$ is a given parameter. More formally:

Definition 1 Given the planning horizon $W$ with length $|W|$ and the set $O$ of $|O|$ caregivers, a set of tours $\mathcal{C}$, with cardinality $|W||O|$, is a complete set of tours if it includes all the certain and all the uncertain requests. A tour in $\mathcal{C}$ is called a complete tour.

Given a complete set of tours $\mathcal{C}$, for any tour $\tau \in \mathcal{C}$, let $\bar{N}(\tau)$ and $\tilde{N}(\tau)$ be the subsets of certain and uncertain nodes in the tour, respectively.

Definition $2 A$-tour of $\tau$ is a tour composed of all the nodes in $\bar{N}(\tau)$ and no more than $\Gamma$ nodes in $\tilde{N}(\tau)$.

We say that a complete tour $\tau$ generates a set of $\Gamma$-tours. These $\Gamma$-tours differ for the subset of (at most $\Gamma$ ) uncertain nodes of $\tau$ that they include. Figure 1 shows an example of a complete tour $\tau$ - Fig. 1(a), and a subset of $\Gamma$-tours generated from it, for $\Gamma=0,1,2$ - Fig. 1(b)-(h). The dark nodes are the certain ones, while the gray nodes are uncertain. $R$ denotes the length, or workload, of each tour, and it is composed both of service times at patients - 45 minutes for each visit- and routing times reported on the arcs. Note that, with a workday length fixed to 240 , the most conservative scenario where all the uncertain requests are scheduled - Fig. 1(a) would be infeasible. In contrast, each generated $\Gamma$-tour - Fig. 1(b)-(h) - would be feasible.

Now we introduce the proposed concept of Home Care robustness by considering a specific subset of the $\Gamma$-tours, according to the following definition:

Definition $3 A$ sequence-preserving $\Gamma$-tour is a $\Gamma$-tour whose nodes represent a subsequence of the complete tour that generated it.

In other words, a sequence-preserving $\Gamma$-tour is a particular type of $\Gamma$-tour derived from the complete tour by deleting some uncertain nodes, without changing the order of the remaining ones. Notice that sequence-preserving $\Gamma$-tours are significant from an operational perspective, since caregivers may not want to alter the order of the patient visits, planned via the complete set of tours, in case some uncertain requests do not realize or are not confirmed on a daily basis. 


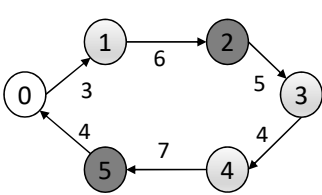

(a) Complete tour $\mathrm{R}=254>240$

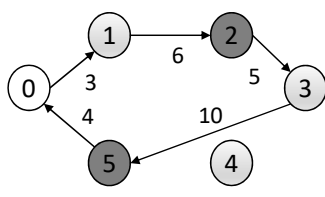

(b) $\Gamma=2$

$\mathrm{R}=3+6+5+10+4+180=208$

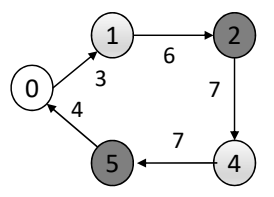

(3)

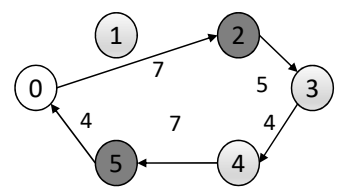

(c) $\Gamma=2$

$\mathrm{R}=3+6+7+7+4+180=207$ (d) $\Gamma=2$

$\mathrm{R}=7+5+4+7+4+180=207$

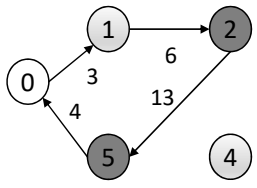

(3)

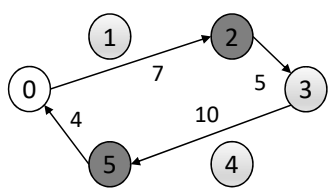

(f) $\Gamma=1$

$\mathrm{R}=3+6+13+4+135=161$
$\mathrm{R}=7+5+10+4+135=161$

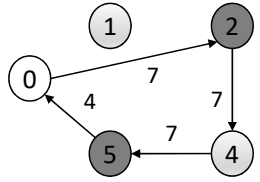

(3)

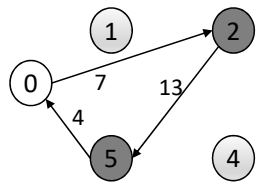

(g) $\Gamma=1$

$\mathrm{R}=7+7+7+4+135=160$ (h) $\Gamma=0$

$\mathrm{R}=7+13+4+90=114$

Figure 1: A complete tour and some generated $\Gamma$-tours

Definition 4 A complete set of tours $\mathcal{C}$ is a robust set of tours with respect to $\Gamma$ if, for each tour $\tau \in \mathcal{C}$, the length of each sequence-preserving $\Gamma$-tours generated by $\tau$ does not exceed the workday length of the caregiver assigned to $\tau$.

According to the definition above, $\mathcal{C}$ is a robust solution, with respect to $\Gamma$, if for each tour $\tau \in \mathcal{C}$, the caregiver assigned to $\tau$ is able to perform the tour whatever the (at most $\Gamma$ ) uncertain requests of $\tau$ will possibly realize, without changing the order of the remaining visits. Equivalently, the maximum length of the sequencepreserving $\Gamma$-tours generated by $\tau$ must be less than or equal to the workday length of the caregiver. Hereafter each sequence-preserving $\Gamma$-tour showing the maximum length will be referred to as a critical $\Gamma$-tour of $\tau$. For example, the $\Gamma$-tours in Figure 1 are the sequence-preserving $\Gamma$-tours for the represented complete tour, for $\Gamma=0,1,2$, respectively. By recalling that $R$ denotes the length of each tour, then the $\Gamma$-tour in (b) is the critical one for $\Gamma=2$, while the ones in (e) and (f) are critical for $\Gamma=1$. Notice that, if the complete tour is assigned to a caregiver with workday length 240, the assignment turns out to be feasible, according to the considered form of robustness, for $\Gamma=0,1,2$. In fact the length of each critical tour (and, consequently, of each sequence-preserving $\Gamma$-tour) is less than or equal to 240 . 
Based on this form of robustness, the sequence-preserving $\Gamma$-Robust Home Care Problem, or $s R H C_{\Gamma}$ for short, consists in jointly addressing:

- care plan scheduling: assigning a pattern to each patient $j$, and a pattern to its uncertain "copy" $j+n$, so scheduling all his certain requests, expressed by $\bar{r}_{j}$, and all his uncertain requests, expressed by $\tilde{r}_{j}$, during the planning horizon;

- caregiver assignment: assigning caregivers to each patient $j$ and to the uncertain "copy" $j+n$, for each day where a request, respectively certain and uncertain, has been scheduled;

- daily caregiver routing: determining the tour of each caregiver for each scheduled day, so that the resulting complete set of tours constitutes a robust set of tours with respect to $\Gamma$.

As for the nominal case, the skill constraints and the continuity of care have to be taken into account. Furthermore, as in HCP, the objective function guiding the three groups of decisions aims at balancing the caregiver workload. This will be better specified in 3.2.3.

We want to remark that, although an upper bound is put on the number of admitted uncertain events in any feasible robust solution, in line with the cardinalityconstrained robust framework in [3], the proposed form of Home Care robustness is not a pure application of the cardinality-constrained robust framework, indeed. In fact, by exploiting the typical structure of the vehicle routing solutions, i.e. a set of tours, the upper bound $\Gamma|O||W|$ is equally partitioned among the tours (at most $\Gamma$ uncertain requests per tour are in fact admitted), in an attempt to balance the uncertainty among the caregivers, thus providing more balanced working conditions also in uncertain scenarios. The robust framework in [3] is therefore generalized in an uncertainty balancing perspective.

\subsubsection{A robust mathematical model}

In order to model $s R H C_{\Gamma}$, with a little abuse of notation we use $N=\{1, \ldots, 2 n\}$ and $A$ to denote, respectively, the set of patient nodes and the set of the arcs of the enlarged complete logistic network. A "+" beside $N$ or one of its subsets (e.g., $\bar{N}^{+}$) will be used to indicate that we are including node 0, i.e. the depot. Again, with a little abuse of notation, we use $r_{j}$ and $P_{j}$ to denote both the certain and the uncertain care plans and pattern sets, respectively. The distinction between certain and uncertain being provided by the patient index $j$.

Let us define the following families of variables in order to model the care plan scheduling, the caregiver assignment and the routing decisions:

$$
z_{j p}=\left\{\begin{array}{l}
1 \text { if pattern } p \text { is assigned to } j \quad j \in N, p \in P_{j} \\
0 \text { otherwise }
\end{array}\right.
$$


$u_{\omega j}=\left\{\begin{array}{l}1 \text { if caregiver } \omega \text { is assigned to } j \\ 0 \text { otherwise }\end{array} \omega \in O, j \in N\right.$

$x_{i j}^{\omega d}=\left\{\begin{array}{l}1 \text { if caregiver } \omega \text { travels along }(i, j) \text { on day } d \\ 0 \text { otherwise }\end{array}(i, j) \in A, d \in W, \omega \in O_{d}\right.$

Furthermore:

$y_{i j}^{d} \geq 0 \quad$ auxiliary flow variable, which represents the number of nodes visited after $i$ by a caregiver moving along $(i, j)$ on day $d, d \in W$.

Using the variables and notation above, the feasible set of $s R H C_{\Gamma}$ can be modeled as follows:

$$
\begin{array}{ll}
\sum_{(i, j) \in A} \sum_{\omega \in O_{d}} x_{i j}^{\omega d} \leq \sum_{p \in P_{j}: p(d) \geq 1} z_{j p} & \forall j \in N, \forall d \in W \\
\sum_{(i, j) \in A} \sum_{\omega \in O_{d}: s_{\omega} \geq k} x_{i j}^{\omega d} \geq \sum_{p \in P_{j}: p(d)=k} z_{j p} & \forall j \in N, \forall d \in W, \forall k \in K \\
\sum_{p \in P_{j}} z_{j p}=1 & \forall j \in N \\
\sum_{\omega \in O} u_{\omega j} \leq T & \forall j \in N \\
x_{i j}^{\omega d} \leq u_{\omega j} & \forall(i, j) \in A, \forall j \in N, \forall d \in W, \forall \omega \in O_{d} \\
u_{\omega j} \leq \sum_{(i, j) \in A} \sum_{d \in W} x_{i j}^{\omega d} & \forall j \in N, \forall \omega \in O \\
R_{S \Gamma}^{\omega d} \leq D_{\omega} & \forall d \in W, \forall \omega \in O_{d} \\
\sum_{(i, j) \in A} x_{i j}^{\omega d}=\sum_{(j, i) \in A} x_{j i}^{\omega d} & \forall j \in N, \forall d \in W, \forall \omega \in O_{d} \\
\sum_{(0, j) \in A} y_{0 j}^{d}=\sum_{j \in N} \sum_{p \in P_{j}: p(d) \geq 1} z_{j p} & \forall d \in W \\
\sum_{(i, j) \in A} y_{i j}^{d}-\sum_{(j, i) \in A} y_{j i}^{d}=\sum_{p \in P_{j}: p(d) \geq 1} z_{j p} & \forall j \in N, d \in W \\
y_{i j}^{d} \leq 2 n \sum_{\omega \in O_{d}} x_{i j}^{\omega d} & \forall(i, j) \in A, d \in W
\end{array}
$$

Constraints (1) state that at most one caregiver per day can visit $j$, if a visit has been scheduled on that day for node $j$. Constraints (2) guarantee that, on day $d$, for each skill $k$ exactly one caregiver, of adequate skill, must visit $j$ if a service of that skill has been scheduled for $j$ on day $d$. In particular, if two skill levels are considered, caregivers of skill 1 can perform only visits of skill 1 , whereas caregivers of skill 2 can perform both types of visits. Constraints (3) ensure that exactly one pattern is assigned to each patient. Notice that a pattern is assigned to each patient and also to its uncertain copy, therefore we schedule all the certain 
and all the uncertain requests of each patient. The design variables $x_{i j}^{\omega d}$ thus model a complete set of tours according to Definition 1. Constraints (4) express the care continuity requirement, i.e. they bound the number of caregivers that can visit each patient during the time horizon. Constraints (5) guarantee that a caregiver can visit a patient only if assigned to the patient (linking between routing and assignment variables). Furthermore, constraints (6) force $u_{\omega j}$ to zero if caregiver $\omega$ never visits patient $j$ during the week. Constraints (8) are the classical flow conservation constraints on the routing variables. Constraints (9) and (10) are the flow conservation constraints on the auxiliary $y$ variables, which are introduced to avoid subtours. They also guarantee the correct linking between scheduling and flow variables. Constraints (11) link together routing and flow variables.

Finally, constraints (7) are introduced to guarantee that the complete set of tours described by the design variables $x_{i j}^{\omega d}$ is a robust set of tours with respect to $\Gamma$ (see Definition 4). More precisely, for each day $d$ and each caregiver $\omega \in O_{d}$, $R_{S \Gamma}^{\omega d}$ represents the maximum length amongst all the sequence-preserving $\Gamma$-tours generated by the complete tour of $\omega$ on day $d$, say $\tau$. That is, it is the workload of a critical $\Gamma$-tour of $\tau$, as previously defined. By imposing that such a maximum workload cannot exceed the workday duration of $\omega$, we ensure that all the sequencepreserving $\Gamma$-tours generated by $\tau$ satisfy the daily length of $\omega$. Thus, the solution represents a robust set of tours, as required.

\subsubsection{The robust approach}

$s R H C_{\Gamma}$ is a very complex problem, since it involves assignment, scheduling and routing decisions, plus uncertainty of the patient requests. Therefore, we start our study by proposing a matheuristic approach to $s R H C_{\Gamma}$ rather than an exact solution method. This is achieved by replacing each $R_{S \Gamma}^{\omega d}$ expression, in constraints (7), by a suitable upper approximation.

Given a tour $\tau$, let $A(\tau)$ denote the set of $\operatorname{arcs}$ of $\tau$, while $w l(\tau)$ denote its workload. Furthermore, let $t_{j}^{\max }=\max _{(i, j) \in A}\left\{t_{i j}\right\}$ be the maximum travel time amongst all arcs entering $j$, for each node $j$. Then:

Proposition 1 Given a complete tour $\tau$ and a sequence-preserving $\Gamma$-tour $\pi$ generated from $\tau$, the following expression is an upper bound on the workload of $\pi$ :

$$
U(\pi)=\sum_{(i, j) \in A(\pi) \cap A(\tau)}\left(t_{i j}+a_{j}\right)+\sum_{(i, j) \in A(\pi):(i, j) \notin A(\tau)}\left(t_{j}^{\max }+a_{j}\right)
$$

Proof: By definition, the workload of $\pi$ is $w l(\pi)=\sum_{(i, j) \in A(\pi)}\left(t_{i j}+a_{j}\right)$. Now, look at the expression $U(\pi)$. For each arc $(i, j) \in A(\pi)$, two cases happen. Either $(i, j) \in A(\tau)$, too, and in such a case the travel time considered in $U(\pi)$ is the exact value $t_{i j}$. Or $(i, j) \notin A(\tau)$. In this latter case, which verifies when the arc entering 
$j$ in the tour $\tau$ has been removed when generating $\pi$, the exact travelling time $t_{i j}$ is over-estimated by $t_{j}^{\max }$ in $U(\pi)$. Therefore $U(\pi) \geq w l(\pi)$.

The motivation for distinguishing between the $\operatorname{arcs}$ in $A(\pi) \cap A(\tau)$, and the rest, is that the former are fully characterized within the mathematical model by the design variables $x_{i j}^{\omega d}$, and their contribution to the workload of a sequence-preserving $\Gamma$ tour $\pi$ can then be expressed using such a characterization. Indeed, to further simplify the upper bound computation, hereafter only the arcs in $A(\pi) \cap A(\tau)$ that are incident to at least one certain node will be evaluated exactly, i.e. via the values $t_{i j}$, whereas the travel times of the others, i.e. the ones whose end nodes are both uncertain, will be over-estimated, too, via the values $t_{j}^{\max }$.

Now let us formalize the problem of estimating the workload of each critical sequence-preserving $\Gamma$-tour according to the just stated upper approximation. To this end, let us introduce auxiliary decision variables $v_{j}^{\omega d}, \forall j \in \tilde{N}, d \in W, \omega \in O_{d}$. These are binary variables taking on value 1 if the uncertain node $j \in \tilde{N}$ is visited on day $d$ by caregiver $\omega$ (i.e., $j$ belongs to a sequence-preserving $\Gamma$-tour of $\omega$ on day d), 0 otherwise:

$$
v_{j}^{\omega d}=\left\{\begin{array}{l}
1 \text { if } j \text { is visited by caregiver } \omega \text { on day } d \quad j \in \tilde{N}, d \in W, \omega \in O_{d} . \\
0 \text { otherwise }
\end{array}\right.
$$

Using the variables above, for each day $d$ and each caregiver $\omega \in O_{d}$ we study the following upper approximation of $R_{S \Gamma}^{\omega d}$ :

$$
\begin{aligned}
& \bar{R}_{S \Gamma}^{\omega d}=\sum_{(i, j) \in A: i, j \in \bar{N}^{+}}\left(t_{i j}+a_{j}\right) \cdot x_{i j}^{\omega d}+\sum_{(i, j) \in A: i \in \tilde{N}^{\prime}, j \in \bar{N}^{+}}\left(t_{j}^{\max }+a_{j}\right) \cdot x_{i j}^{\omega d}+ \\
& \max \left\{\sum_{(i, j) \in A: i \in \bar{N}^{+}, j \in \tilde{N}}\left(t_{i j}+a_{j}\right) \cdot x_{i j}^{\omega d} \cdot v_{j}^{\omega d}+\sum_{(j, i) \in A: i \in \bar{N}^{+}, j \in \tilde{N}}\left(t_{j i}-t_{i}^{\max }\right) \cdot x_{j i}^{\omega d} \cdot v_{j}^{\omega d}+\right. \\
& \left.\sum_{(i, j) \in A: i \in \tilde{N}, j \in \tilde{N}}\left(t_{j}^{\max }+a_{j}\right) \cdot x_{i j}^{\omega d} \cdot v_{j}^{\omega d}\right\} \\
& \sum_{j \in \tilde{N}} v_{j}^{\omega d} \leq \Gamma \\
& v_{j}^{\omega d} \in\{0,1\} \quad \forall j \in \tilde{N} .
\end{aligned}
$$

Given a complete tour $\tau$, modelled by the $x_{i j}^{\omega d}$, and a sequence-preserving $\Gamma$ tour $\pi$ generated from it, whose uncertain selected nodes are defined by (14) - (15), 
the objective function in (13) estimates the workload of $\pi$ by considering exactly the contribution due to the arcs linking certain nodes as well as the depot (first summation in (13)), and also the contribution of the arcs linking certain nodes and depot to selected uncertain copy nodes, i.e. to nodes $j \in \tilde{N}$ such that $v_{j}^{\omega d}=1$ (third and fourth summation in (13)). Note that all such arcs belong to $A(\tau)$. On the other hand, for the $\operatorname{arcs}(i, j) \notin A(\tau)$, and for the arcs in $A(\tau)$ linking selected uncertain nodes, the traveling time of $(i, j)$ is estimated from above via $t_{j}^{\max }$. Therefore, problem (13)-(15) looks for a critical sequence-preserving $\Gamma$-tour based on the just stated over-estimation of the tour workload. From Proposition 1 we get $\bar{R}_{S \Gamma}^{\omega d} \geq R_{S \Gamma}^{\omega d}$.

The model obtained from (1) - (11) by replacing constraints (7) with:

$$
\bar{R}_{S \Gamma}^{\omega d} \leq D_{\omega} \quad \forall d \in W, \forall \omega \in O_{d}
$$

thus represents a matheuristic to $s R H C_{\Gamma}$. The following property can be easily proved [33]:

Proposition 2 For each day $d$ and each caregiver $\omega \in O_{d}$, the constraint matrix of model (13) - (15) is totally unimodular (TU).

Therefore, by treating the design variables $x_{i j}^{\omega d}$ as constants, a standard technique in Robust Optimization, each inner ILP model (13) - (15) can be replaced by its Linear Programming relaxation and so, by strong duality, by its LP dual, leading to a MILP formulation to the proposed $s R H C_{\Gamma}$ approximation. The complete model is reported in Appendix A.

\subsubsection{Guiding the Home Care decisions}

In the computational experience described in Section 4 we have addressed a social equity criterion, aiming at balancing the caregiver workloads. This kind of criterion was also investigated in [7] and [34]. Precisely, we have considered the objective function minmax, which minimizes the maximum caregiver utilization factor. In the robust framework under study, the caregiver utilization factor is expressed as the total workload of the caregiver during the planning horizon in the worst scenario, i.e. by considering his critical tours, over his maximum possible workload. The corresponding MILP model is thus (constraint numbers refer to Appendix A):

$\min m$

$$
\frac{\sum_{d \in W} \bar{R}_{S \Gamma}^{\omega d}}{|W| \cdot D_{\omega}} \leq m, \quad \forall \omega \in O .
$$


Notice that the alternative balancing objective function, which maximizes the minimum caregiver utilization factor, cannot be used in the same form investigated in [7] and [34], since the corresponding optimal value is always 1 . This is formally proved in Appendix B to this paper.

\subsubsection{Estimating the matheuristic approximation}

To conclude, let us provide an estimate of the absolute error which is committed, under the objective function minmax, when solving the proposed matheuristic approach rather than the exact one, which considers the exact workload of the critical tours in (17) instead of the studied approximation $\bar{R}_{S \Gamma}^{\omega d}$. The analysis will be performed by considering the set of the patients as partitioned into clusters. In real Home Care instances, in fact, the set of the patients is usually clusterised. Precisely, patients located in the same municipality form a cluster of nodes, such that the distance between any pair of nodes within the cluster is very small compared to the distance between nodes belonging to different clusters. Such intra-cluster distances can be usually assumed equal to a certain (small) value $\delta$. In addition, the inter-cluster distances depend only on the clusters, and not on the specific patients within the clusters. This is the case of the Palliative Home Care data set we used in Section 4 , where the set of the patients is organized into 5 or 8 clusters. Note that to consider a cluster organization of the patients is not a loss of generality, indeed, since data sets where this kind of structure is not present correspond to a cluster organization with clusters of cardinality one.

By exploiting the patient's organization into clusters, in our experiments we used the following inequalities, proposed in [7], to take under control the workday of the operators in a computationally efficient way:

$$
\sum_{i \in(N \backslash C)} \sum_{j \in C} x_{i j}^{\omega d} \leq 1 \quad \forall C, \forall d \in W, \forall \omega \in O_{d} .
$$

(18) impose that, for each cluster of patients $C$, and in each day of the planning horizon, each available operator enters $C$ at most once. These cuts, referred to as Exploit-Cluster, or EC, model a reasonable requisite in the studied Home Care problem, since in the absence of time windows associated with patient visits, stakeholders usually require that operators visit each municipality at most once per day.

Below we provide an estimate of the error committed by the matheuristic when (18) are used to enhance the approach. The estimate relies on the observation that, due to the distance structure, any permutation of a subset of patients belonging to the same cluster induces the same identical workload contribution to the objective function. In the expression, $N_{c}$ denotes the number of clusters, $T_{c}^{\text {max }}$ and $T_{c}^{\text {min }}$ are the maximum and the minimum inter-cluster distance, respectively. For the proof 
of the statement we refer to Appendix $\mathrm{C}$ to this paper.

Proposition 3 For any feasible solution, the absolute error committed by the matheuristic approach, under the objective function minmax, with respect to the objective function value of $s R H C_{\Gamma}$ is bounded from above by:

$$
|W||O|\left(N_{c}\left(T_{c}^{\max }-T_{c}^{\min }\right)+(\Gamma-1)\left(T_{c}^{\max }-\delta\right)\right) .
$$

Therefore:

Corollary 1 The absolute error committed by the matheuristic approach, under the objective function minmax, with respect to the optimal value of $s R H C_{\Gamma}$ is bounded from above by $|W \| O|\left(N_{c}\left(T_{c}^{\max }-T_{c}^{\text {min }}\right)+(\Gamma-1)\left(T_{c}^{\text {max }}-\delta\right)\right)$.

Note that, according to the stated result, the error committed by the proposed upper approximation can be small when the number of the caregivers is limited, and the addressed Home Care instances are characterised by a few, and quite dense, clusters. This is the case of the data set investigated in Section 4, where $|W|=5$, and the number of the clusters is either 5 or 8 . Clearly, the error also depends on the robustness parameter $\Gamma$, which is however a constant for the considered approach.

\section{Computational experiments}

We report the results of some experiments on Robust Home Care instances, which have been generated starting from some benchmarks in the literature. The aim has been to assess the efficiency of the sequence-preserving $\Gamma$-Robust Home Care approach, proposed in this paper, the quality of the returned solutions and their degree of robustness. Also, we wanted to have some indications on the characteristics of the instances which can be affordably addressed via the presented robust approach.

Traditionally, the efficiency and the efficacy of robust approaches are investigated via the so-called "Price of Robustness". That is, by estimating the price that we are willing to pay to guarantee robustness, using robust solutions instead of the "cheaper" nominal ones [19], which may become unfeasible when some uncertain events realize. In the Home Care context under study, instead, the situation is radically different. In fact, since each nominal solution only takes into account certain requests, it becomes unfeasible when uncertain requests realize. In order to take into account such realized requests, the computed nominal solutions should be modified by scheduling the new requests over the time horizon, and inserting them into some caregiver tours, provided that the skill constraints and the workday capacity constraints remain satisfied. But, as the results presented in the rest of this section reveal, also for instances apparently of small size, with 40 patients and 
2 caregivers, computing nominal solutions may be as difficult as determining robust solutions. Therefore, it may not be convenient to invest in the nominal solution computation, and then try to modify the found solutions, if the robust approach returns solutions of good quality and degree of robustness with about the same computational effort.

The validity of this kind of assessment is corroborated by the fact that, as shown in Section 4.5, if we fix the scheduling decisions by assigning a pattern in $P_{j}$ to each patient $j$ (i.e. we fix the decision variables $z_{j p}$ in constraints (3)), so decomposing the robust approach along the lines outlined in [34], then the sequence-preserving $\Gamma$ Robust Home Care approach proves to gain a lot in terms of computational efficiency, still returning robust solutions of good quality. Therefore, it appears to be a solid tool to face with instances of reasonable size, and a basic block to address instances of larger size, via decomposition approaches. The latter aspect, preliminary assessed in this paper, is the subject of current investigation.

The main achievements that are shown can be summarized as follows:

1. the proposed matheuristic approach is able to efficiently address Robust Home Care instances of moderate size, in terms of number of patients and caregivers, by returning solutions near to the optimal ones, and the efficiency does not deteriorate too much as parameter $\Gamma$ increases; note that, despite the moderate size, such instances as well as their nominal counterparts are difficult to address;

2. the computed solutions are well-balanced, i.e. the average percentage difference between the maximum and the minimum caregiver utilization factor $\left(\operatorname{Avg} \Delta_{U F}\right)$ is very small; concerning the number of uncertain requests that are selected in the critical tours, this number varies greatly, and tends to increase with $\Gamma$;

3. the proposed upper approximation of the caregiver utilization factor is a good estimate, since a small increase of the objective function value is empirically testified;

4. the degree of robustness of the computed solutions, experimented on a subset of scenarios, and measured in terms of percentage of uncertain visits that can be scheduled in case they realize, is significant and tends to increase with $\Gamma$;

5. by fixing the scheduling decisions, the efficiency of the approach drastically improves, with a very small deterioration of the quality of the returned solutions in terms of $\operatorname{Avg} \Delta_{U F}$ and approximation error; on the contrary, the number of the selected uncertain requests increases; therefore, this kind of decomposition seems to be suitable to successfully address robust instances of larger size. 


\subsection{The robust data set}

The robust instances have been generated starting from the data set used in [7] and in [34], which is publicly available at http://www.di.unipi.it/optimize/.

It comprises real world Clusterised Home Care instances provided by one of the largest Italian public medical care unit, on a 5-day planning horizon. These instances are characterized by 2 types of hierarchical skills, travelling times obtained via Google Maps, and a service time at the patients equal to 45 minutes. Specifically, we selected 12 source instances among the smaller ones in the data set. Each source instance refers to a week in January 2006, and it is characterized by 40 or 60 patients organized in 5 or 8 clusters or municipalities ( 4 combinations). Furthermore, there are 3 instances, namely 0,1 or 2 , for each combination.

From each source instance, 9 robust instances have been generated by considering the combinations of:

- $\alpha$, that represents the percentage of the nodes of the source instance which are considered certain $(0.7,0.8$ and 0.9$)$,

- 3 random seeds for generating the uncertain requests ( $A, B$ and $C$ hereafter),

thus summing up to 108 robust instances. Each instance is thus identified by a string reporting the following fields separated by a "“" character: the week, the number of municipalities, the number of patients, the instance identifier in the group (i.e. 0,1 or 2 ), the value of $\alpha$ and the seed (i.e. $A, B$ or $C$ ).

With respect to the source instances, the robust instances with 40 patients are characterized by one caregiver of skill 1 with a workday of 8 hours, and one caregiver of skill 2 with a workday of 6 hours. On the other hand the robust instances with 60 patients have an additional caregiver of skill 1 (therefore, 2 caregivers of skill 1 , and 1 caregiver of skill 2). The reduction of the number of the caregivers in the robust instances is motivated by the fact that, in the robust scenarios, only a subset of the requests, i.e. the certain ones, must be covered necessarily. Moreover, the source instances exhibited a quite low utilization factor for the caregivers. Finally, for each robust instance the care continuity has been set to 1 (i.e. $T=1$ ), while the patterns have been generated by using the flow based method proposed in [7].

The experiments have been performed on an Intel Xeon E5501 $2.00 \mathrm{GHz}$ with 4 processors using Cplex 12.3. The time limit has been set to 12 hours (43.200 seconds), while the memory limit is 1 Gbyte.

\subsection{Assessing the efficiency of the approach}

The analysis will be performed separately for the robust instances with 40 patients and for the larger ones, with 60 patients. For each robust instance both the nominal counterpart, i.e. the instance obtained by deleting all the uncertain requests, and 


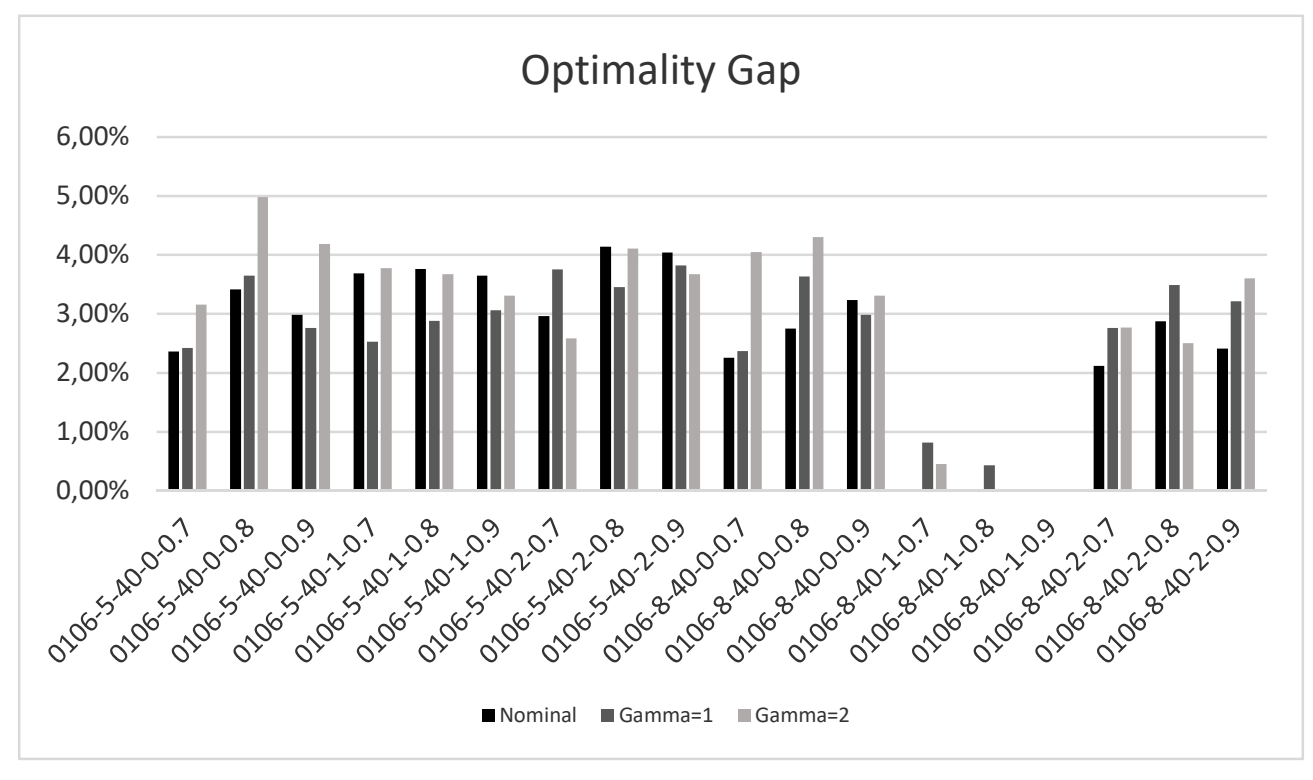

Figure 2: 40 patients - optimality gap

the more conservative counterpart, i.e. the instance obtained by treating all the uncertain requests as certain, have been solved, in order to better assess the impact of the considered levels of robustness. Specifically, $\Gamma=1$ and $\Gamma=2$ have been analysed for the robust instances with 40 patients, whereas $\Gamma=1$ has been investigated for the instances with 60 patients. The figures related to the more conservative counterparts are not reported since for all of them the solver certified the instance unfeasibility.

\subsubsection{Instances with 40 patients}

The graph in Figure 2 reports the average optimality gaps, i.e. the average values, across the seed, for the percentage relative gap with respect to the best lower bound obtained in the branch and bound tree, while the one in Figure 3 shows the average computational time (in seconds).

Observe that, although both the nominal and the robust instances appear difficult to solve, the returned optimality gaps are quite small, especially for the configuration with 8 clusters. In particular, the robust approach shows to be able to compute solutions near to the optimal ones. Also observe that there are groups of instances in which the optimality gaps of the solutions provided by the robust model are smaller than the ones returned by the nominal model. For the robust model with 


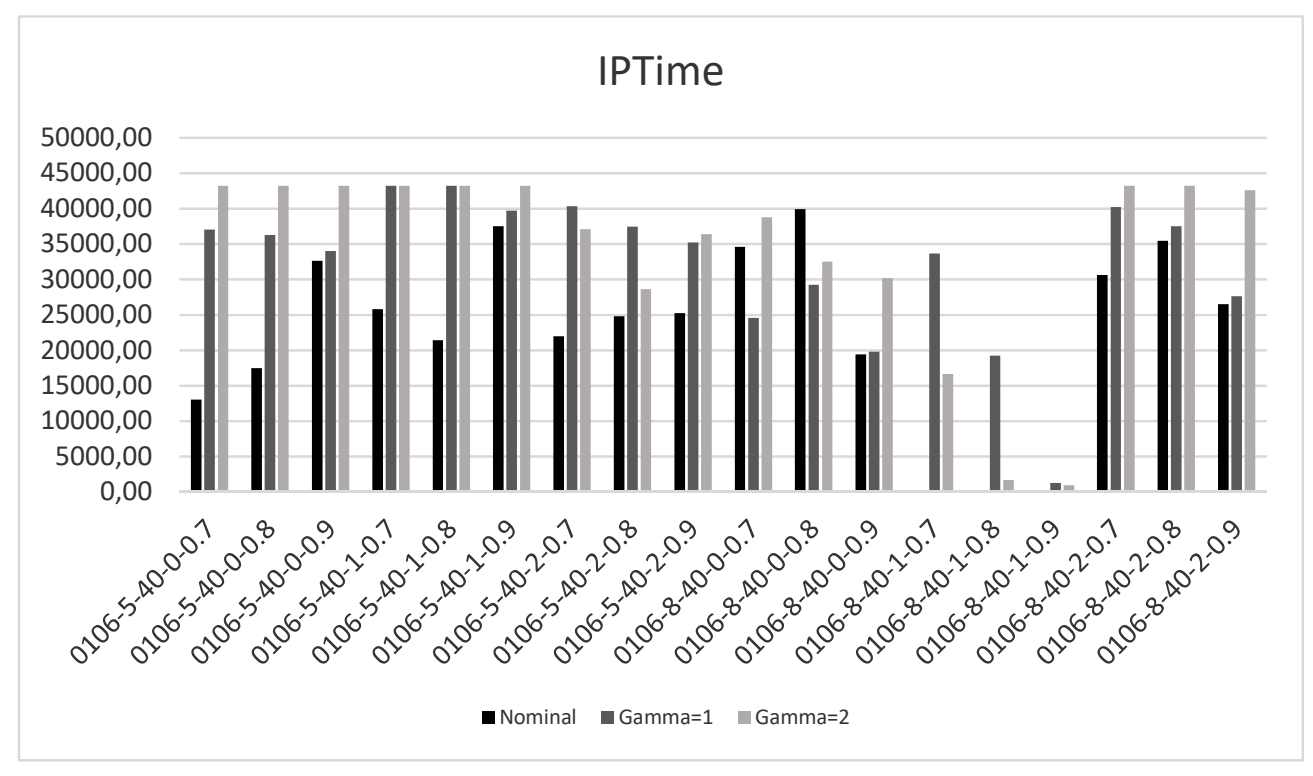

Figure 3: 40 patients - IPTime

$\Gamma=1$, an explanation for this fact may be that, for almost every group of instances, the number of terminations due to a memory limit decreases with respect to the nominal model. This implies that when robustness is considered, the portion of the branch and bound tree explored is greater than in the nominal case.

More in detail, whereas the nominal instances appear to become more difficult when $\alpha$, i.e. the percentage of the certain requests, increases, for the robust approach the case $\Gamma=2$ appears to be computationally harder than $\Gamma=1$. The worst scenario appears to be $\alpha=0.8$ combined with $\Gamma=2$, especially for the instances with 5 clusters. This is quite reasonable, indeed, since this is the scenario where the number of the certain requests, which must be necessarily served, is quite high and, at the same time, the number of the subsets of uncertain requests is significant, so that the number of the sequence-preserving $\Gamma$-tours to be considered in the inner maximization problems may determine an explosion of the problem solution space.

\subsubsection{Instances with 60 patients}

The robust instances with 60 patients proved to be more difficult to address. In particular, within the considered time and memory limit, a solution was found for 17 over 27 instances with 5 clusters, and for 13 over 27 instances with 8 clusters. In 
Table 1: 60 patients: performance metrics (AvgIPTime is in seconds)

\begin{tabular}{lcrr}
\hline Instance & NInst & AvgIPTime & AvgGap \\
\hline $0106-5-60-0-0.7$ & 1 & 43200.00 & $11.61 \%$ \\
$0106-5-60-0-0.8$ & 2 & 43200.00 & $5.62 \%$ \\
$0106-5-60-0-0.9$ & 1 & 43200.00 & $6.71 \%$ \\
\hline $0106-5-60-1-0.7$ & 3 & 43200.00 & $1.11 \%$ \\
$0106-5-60-1-0.8$ & 2 & 2845.00 & $0.00 \%$ \\
$0106-5-60-1-0.9$ & 3 & 19252.67 & $0.19 \%$ \\
\hline $0106-5-60-2-0.7$ & 2 & 43200.00 & $17.98 \%$ \\
$0106-5-60-2-0.8$ & 2 & 43200.00 & $5.59 \%$ \\
$0106-5-60-2-0.9$ & 1 & 43200.00 & $14.94 \%$ \\
\hline $0106-8-60-0-0.7$ & 0 & n.a. & n.a. \\
$0106-8-60-0-0.8$ & 1 & 43200.00 & $6.76 \%$ \\
$0106-8-60-0-0.9$ & 1 & 43200.00 & $8.08 \%$ \\
\hline $0106-8-60-1-0.7$ & 1 & 43200.00 & $12.59 \%$ \\
$0106-8-60-1-0.8$ & 2 & 43200.00 & $10.59 \%$ \\
$0106-8-60-1-0.9$ & 1 & 43200.00 & $5.14 \%$ \\
\hline $0106-8-60-2-0.7$ & 3 & 39937.33 & $3.97 \%$ \\
$0106-8-60-2-0.8$ & 2 & 43200.00 & $16.30 \%$ \\
$0106-8-60-2-0.9$ & 2 & 43200.00 & $6.47 \%$ \\
\hline
\end{tabular}

addition, one instance with 5 clusters and one with 8 clusters proved to be unfeasible. The optimality gap was $5.62 \%$ in the case of 5 clusters (average gap over the 17 feasible instances), and $8.55 \%$ in the case of 8 clusters (average gap over the 13 feasible instances), therefore greater, on average, than the one related to the instances with 40 patients. Table 1 details the obtained results. In this table, column NInst reports the number of instances of each group for which a solution was found, and string "n.a." is used to point out that no feasible solution was found. The other columns report the average computational time, in seconds (AvgIPTime), and the average optimality gap (AvgGap).

\subsection{Assessing the robust solution quality}

Also these results are presented separately for the instances with 40 patients and for those with 60 patients. The quality of the solutions will be given in terms of the metric $\operatorname{Avg} \Delta_{U F}$, number of uncertain requests selected in the critical tours (or equivalently number of $v_{j}$ s variables set to one), and error committed by the matheuristic against the exact approach, estimated theoretically in Section 3.2.4 (see Proposition 3). Precisely, the empirical relative error has been computed by determining, a posteriori, the critical tours (note in fact that the model (20) - (33) 


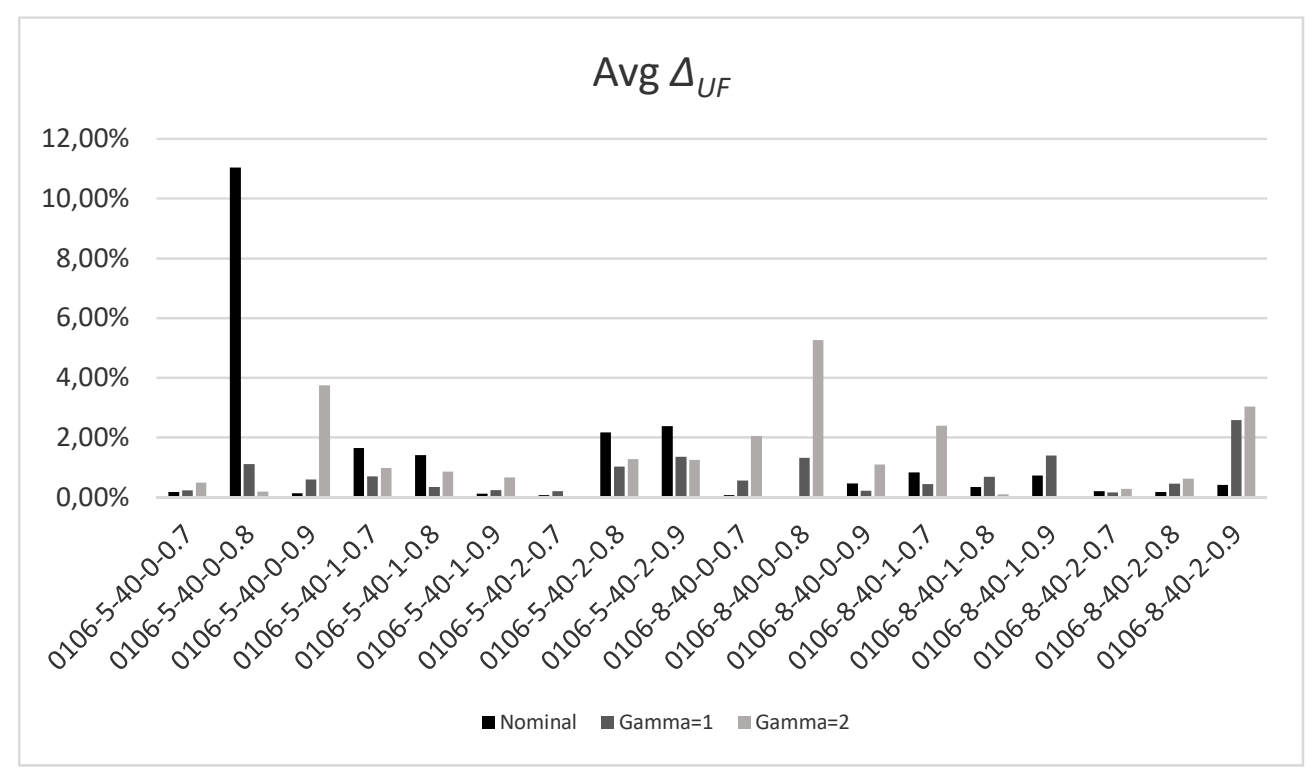

Figure 4: 40 patients - metric $\operatorname{Avg} \Delta_{U F}$

does not return such tours explicitly), and calculating the difference between the upper estimate, addressed by the inner ILP model (13) - (15), and the sum of the corresponding, exact, workloads. To determine the critical tours, the selected uncertain requests of the complete tours must be found; i.e., for each day $d$ and each caregiver $\omega \in O_{d}$, the nodes $j \in \tilde{N}$ such that $v_{j}^{\omega d}=1$. This has been obtained by ordering the uncertain nodes in the complete tour of $\omega$ on day $d$ in a nonincreasing way with respect to their cost in the objective function of (13) - (15) (notice that the design variables $x_{i j}^{\omega d}$ are constant at this level), and selecting the first positive $\Gamma$ ones according to this ordering (or all the positive ones, if their number is less than $\Gamma)$.

\subsubsection{Instances with 40 patients}

Figure 4 reports the metric $\operatorname{Avg} \Delta_{U F}$, i.e. the average percentage difference between the maximum and the minimum caregiver utilization factor. Interestingly, the robust solutions appear to be more equitable than the nominal ones in terms of caregiver utilization factor. This is especially true for the configuration with 5 clusters and for the setting $\Gamma=1$.

Another interesting issue is the quality of the computed solutions in terms of 


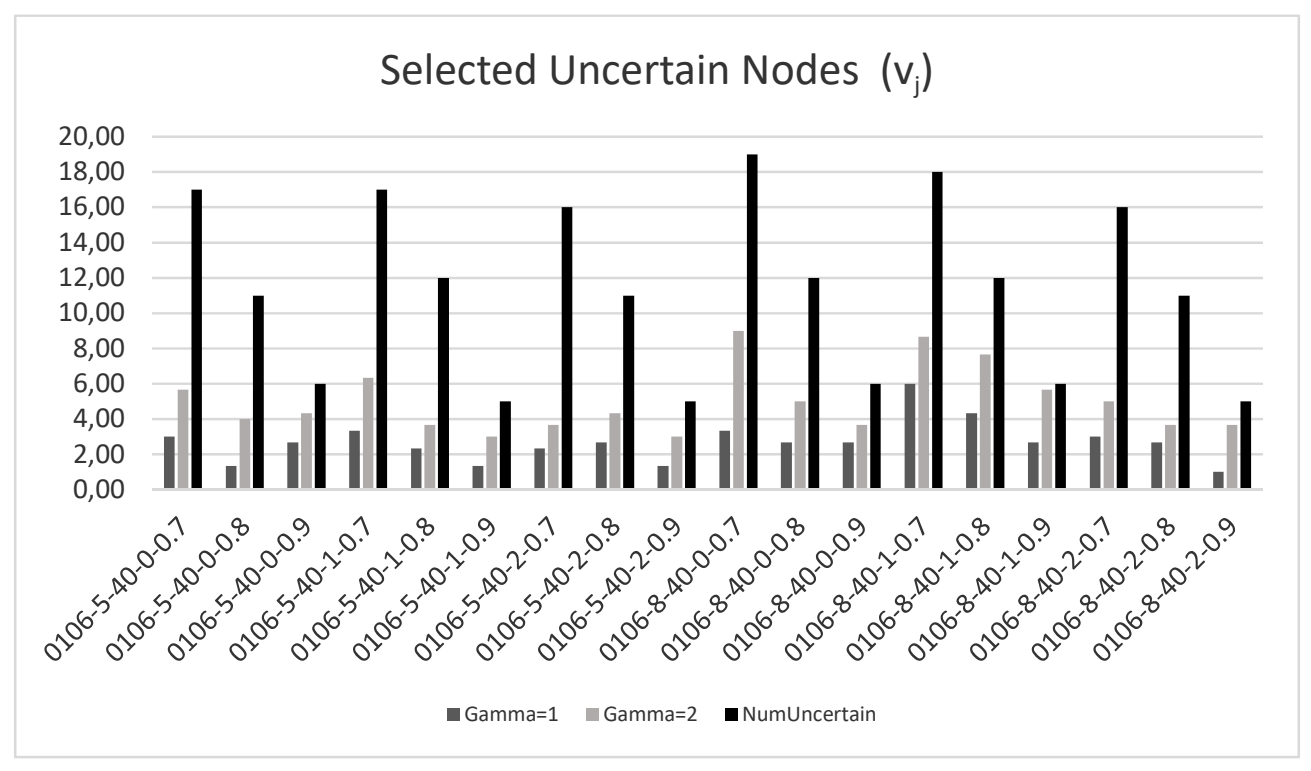

Figure 5: 40 patients - number of uncertain nodes selected in robust solutions

number of uncertain requests that are selected in the robust tours. At this regard, Figure 5 shows this number compared to the total number of uncertain requests. This number, given by the average number of the $v_{j}$ s variables set to one in the provided solutions, varies greatly across the groups of instances and also across the robust instances within the same group. While it is reasonable to assume that, for a given source instance, such a number tends to increase as the number of certain visits decreases (i.e. when $\alpha$ decreases), it clearly depends on the scheduling of the requests along the planning horizon, via the selected patterns, and on the distribution of the uncertain visits across the skills. Figure 5 shows that the average number of selected uncertain requests increases when going from $\Gamma=1$ to $\Gamma=2$, but there are groups of instances for which it remains quite far from the total number of uncertain requests.

By addressing the quality of the proposed matheuristic approximation, Figure 6 reports the empirical percentage relative error committed by the approach. As shown, this error is very small, especially for the configuration with 5 clusters.

Finally, as an additional information we report that, when $\Gamma=1$, the uncertain visits are almost everywhere in charge of the more skilled caregivers, whereas the distribution is more balanced among the caregivers when $\Gamma=2$. Precisely, when 5 municipalities are considered, the number of instances for which both caregivers 


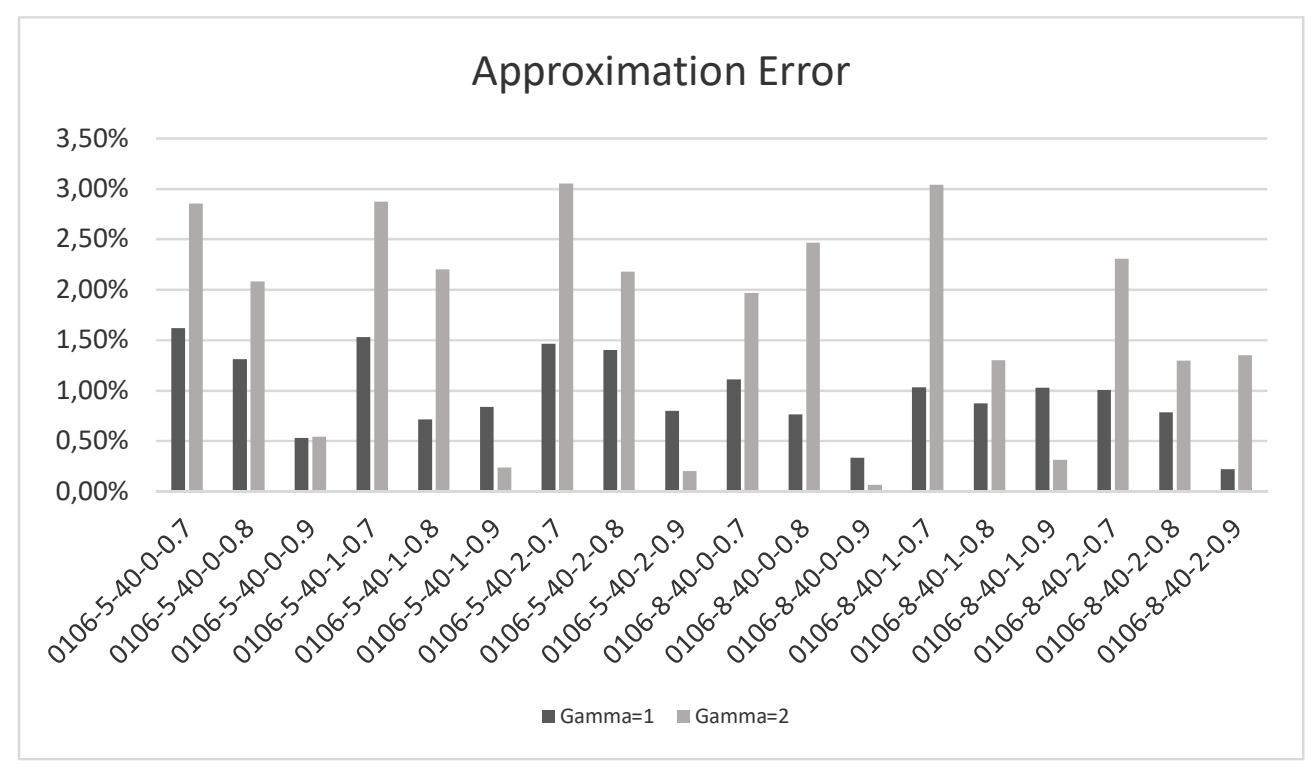

Figure 6: 40 patients - approximation error

are assigned uncertain visits goes from $5 / 27$ for $\Gamma=1$ to $16 / 27$ for $\Gamma=2$. With 8 municipalities, the figures are $17 / 27$ for $\Gamma=1$ and $24 / 27$ for $\Gamma=2$.

\subsubsection{Instances with 60 patients}

Details on the quality of the robust solutions for the instances with 60 patients are given in Table 2. As shown, the average number of uncertain requests that are selected (column $\operatorname{Avg} v_{j}$ ), compared to the total number of uncertain requests (column AvgNInc), varied greatly also in this case. Furthermore, also for these larger instances the average percentage difference between the maximum and the minimum caregiver utilization factor (column $\operatorname{Avg} \Delta_{U F}$ ) proved to be very small, showing a good balancing of the workload among the caregivers.

Concerning the percentage relative error committed by the matheuristic approach (column AvgAErr), the error is very small also for these instances, going from a minimum of $0.05 \%$ to a maximum of $2.97 \%$. In addition, in this scenario we better investigated the quality of the proposed approximation by means of an alternative metric (see column AvgLBErr in Table 2). Differently from AvgAErr, which is related to the approximated inner ILP model (13) - (15), when AvgLBErr is considered, each inner problem is solved exactly, i.e. using exact traveling times. Then, 
Table 2: 60 patients - quality metrics

\begin{tabular}{lrrrrr}
\hline Instance & $\operatorname{Avg} v_{j}$ & AvgNInc & Avg $\Delta_{U F}$ & AvgAErr & AvgLBErr \\
\hline $0106-5-60-0-0.7$ & 8.00 & 25.00 & $1.94 \%$ & $1.55 \%$ & $1.09 \%$ \\
$0106-5-60-0-0.8$ & 3.50 & 16.00 & $1.56 \%$ & $1.34 \%$ & $1.12 \%$ \\
$0106-5-60-0-0.9$ & 3.00 & 8.00 & $0.57 \%$ & $0.47 \%$ & $0.47 \%$ \\
\hline $0106-5-60-1-0.7$ & 9.00 & 22.00 & $1.10 \%$ & $1.95 \%$ & $1.56 \%$ \\
$0106-5-60-1-0.8$ & 6.00 & 15.00 & $1.90 \%$ & $2.20 \%$ & $1.97 \%$ \\
$0106-5-60-1-0.9$ & 2.67 & 7.00 & $0.23 \%$ & $0.55 \%$ & $0.55 \%$ \\
\hline $0106-5-60-2-0.7$ & 7.00 & 23.00 & $5.65 \%$ & $2.97 \%$ & $2.07 \%$ \\
$0106-5-60-2-0.8$ & 2.50 & 16.00 & $0.40 \%$ & $1.37 \%$ & $0.69 \%$ \\
$0106-5-60-2-0.9$ & 4.00 & 7.00 & $6.93 \%$ & $1.11 \%$ & $1.11 \%$ \\
\hline $0106-8-60-0-0.7$ & n.a. & n.a. & n.a. & n.a. & n.a. \\
$0106-8-60-0-0.8$ & 4.00 & 17.00 & $1.64 \%$ & $0.05 \%$ & $0.05 \%$ \\
$0106-8-60-0-0.9$ & 3.00 & 9.00 & $2.44 \%$ & $0.64 \%$ & $0.53 \%$ \\
\hline $0106-8-60-1-0.7$ & 7.00 & 23.00 & $0.86 \%$ & $1.76 \%$ & $0.00 \%$ \\
$0106-8-60-1-0.8$ & 3.50 & 16.00 & $1.73 \%$ & $1.03 \%$ & $1.03 \%$ \\
$0106-8-60-1-0.9$ & 3.00 & 8.00 & $0.63 \%$ & $1.16 \%$ & $1.16 \%$ \\
\hline $0106-8-60-2-0.7$ & 5.00 & 25.00 & $1.01 \%$ & $1.13 \%$ & $1.11 \%$ \\
$0106-8-60-2-0.8$ & 5.00 & 17.00 & $5.51 \%$ & $1.77 \%$ & $1.32 \%$ \\
$0106-8-60-2-0.9$ & 3.00 & 8.00 & $0.41 \%$ & $0.84 \%$ & $0.84 \%$ \\
\hline
\end{tabular}

the percentage relative error between the upper estimate returned by the matheuristic, and the sum of the workloads of the optimal tours so determined, is computed. Note that, for each day and caregiver, the workload addressed in AvgLBErr, being related to the optimal solution of an inner ILP model (13) - (15), is greater than, or equal to, the one of the corresponding critical tour addressed in AvgAErr. Therefore, the estimates in AvgLBErr are more accurate than the ones in AvgAErr. More, since AvgLBErr considers the exact workload of the optimal tours for the given scheduling and assignment decisions, then the difference between the values in AvgAErr and the values in AvgLBErr provides the net error induced by some arc cost over-estimate. Such differences are negligible, often null, so emphasizing the goodness of the proposed upper approximation.

\subsection{Sensitivity analysis and rate of covered uncertain requests}

In this section, we present the results obtained by performing a sensitivity analysis on the value of $\Gamma$ on a small subset of instances. On the same subset we measure the robustness of the generated solutions with respect to possible realizations of uncertain requests. Specifically, we consider the three robust instances generated from the source instances 0106-5-40-0, 0106-5-40-1, and 0106-5-40-2 for $\alpha=0.7$ and 


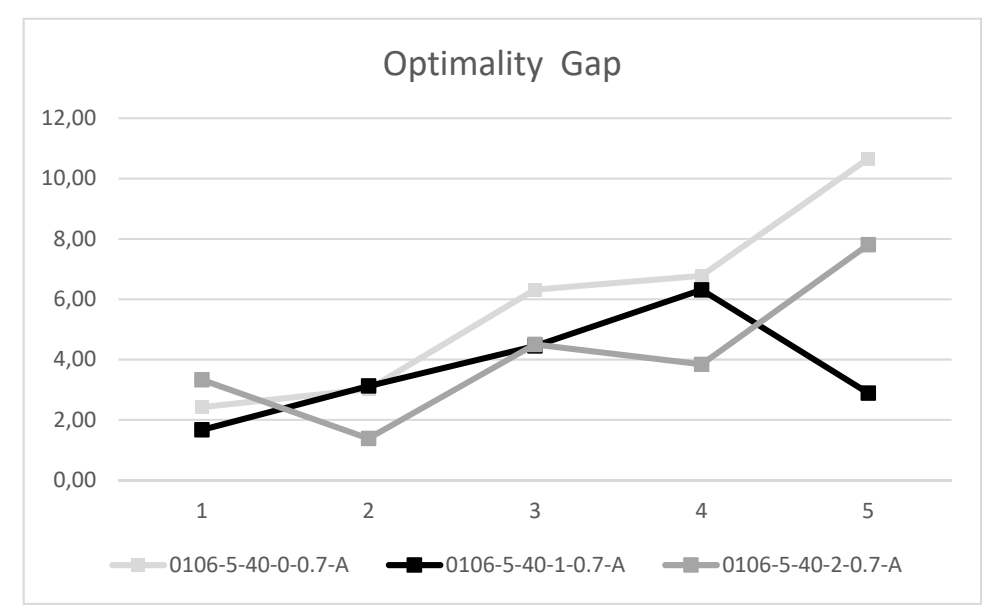

Figure 7: Optimality gap vs $\Gamma$

seed $A$.

Figure 7 reports the optimality gap obtained on the three instances as the value of $\Gamma$ ranges in $\{1, \ldots, 5\}$ with a time limit fixed to $2 \mathrm{CPU}$ hours. Observe that the gap is still acceptable even for quite high values of $\Gamma$. The computational results, even if reported for a small set of instances, seem thus to suggest that our approach is a viable option to address robustness in Home Care.

In order to evaluate the robustness of the generated solutions with respect to possible realizations of uncertain requests, for each robust instance in the set we generated 5 samples of possible scenarios in which a fixed number $(5,10$ or 15$)$ of uncertain requests realize, thus summing up to 45 scenarios. Note that this number can be very close to the total number of uncertain requests that characterize the robust instances (17, 17 and 16). Then, for each realization, we count the number of uncertain visits that can be scheduled according to the robust tours, i.e. we sum the numbers of (at maximum $\Gamma$ ) realized uncertain requests appearing in the robust tours. Figures 8 and 9 report, respectively for $\Gamma=2$ and $\Gamma=3$, averaged on the 5 samples, the percentage of realized uncertain visits that can be scheduled in robust tours with respect to the number of randomly generated uncertain requests (\%CoverVsRandomVisits) and the percentage of realized uncertain visits that can be scheduled in robust tours with respect to the maximum number of uncertain visits allowed by the robust tours (\%CoverVsTourStructure). Observe that \%CoverVsRandomVisits may vary greatly from one instance to the other but it is never below $24 \%$ and it usually reaches satisfactory values. In addition, \%CoverVsRandomVisits increases as $\Gamma$ increases. Thus, since the results in Figure 7 reveal that 


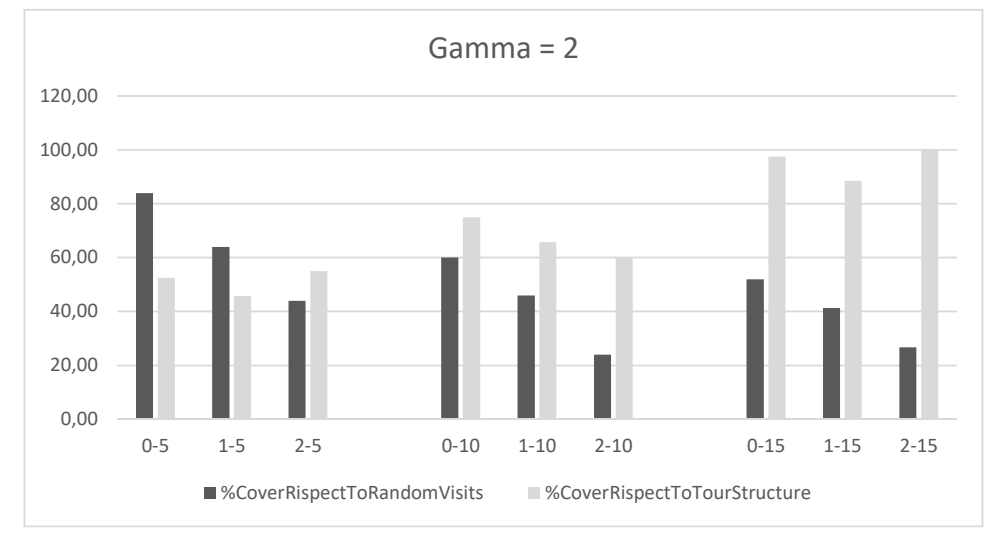

Figure 8: Uncertain requests covered by robust solutions $(\Gamma=2)$

the computational efficiency does not deteriorate too much as the value of $\Gamma$ increases, acting on $\Gamma$ is a good lever to increase robustness. Finally, it is worthwhile to observe that when \%CoverVsRandomVisits is quite low, \%CoverVsTourStructure is however very high, thus suggesting that low rates of scheduled uncertain visits potentially originate from the structure of the tours themselves; indeed, there are some cases where the uncertain requests are concentrated in very few tours. This observation is further corroborated by preliminary results in [8], showing that acting directly on the pattern generation tool and imposing that uncertain visits are quite spread among the robust tours is indeed a smart lever to increase robustness.

\subsection{Fixing the scheduling decisions: a decomposition approach}

The scheduling decisions rely on a set of patterns, which have been generated by using the flow based method in [7]. This method determines a feasible pattern for each patient $j$, that is a pattern which is compatible with the care plan of $j$. To enhance their flexibility, however, both the deterministic and the robust approach allow one to use also patterns of other patients, provided that with the same care plan of $j$ : this defines the pattern subset $P_{j}$ introduced in Section 2 .

In order to gain in efficiency, we experimented a decomposition of the robust approach where each patient $j$ is associated with exactly the pattern which the flow based method has determined for $j$. Therefore, the scheduling decisions are fixed. We performed some preliminary experiments on the subset of the robust instances with 40 patients and $\alpha=0.7$. The obtained results, still preliminary, are very inter- 


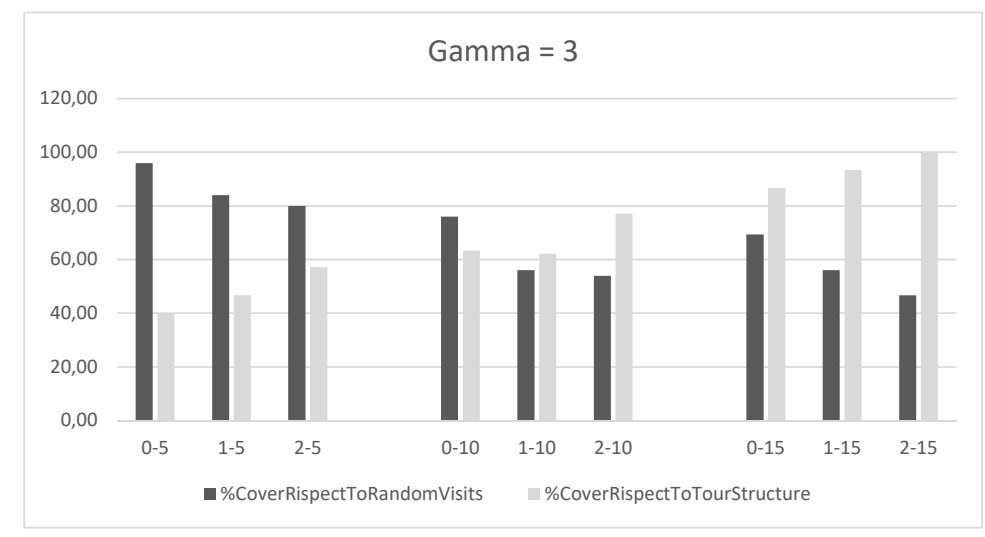

Figure 9: Uncertain requests covered by robust solutions $(\Gamma=3)$

esting. Firstly, the decomposed approach always determined an optimal solution. Second, it was orders of magnitude faster than the robust approach. In fact, it required 468 seconds on average, with a minimum of 9 seconds and a maximum of 2064 seconds.

Furthermore, concerning the quality of the computed robust solutions, only in a few cases the metric $\operatorname{Avg} \Delta_{U F}$ slightly increases (see Figure 10), whereas in the other cases it reduces, by revealing the determination of still more balanced robust solutions. Interestingly, the number of the uncertain requests that are selected by the decomposed approach is greater than in the not decomposed version, as reported in Figure 11. This phenomenon could seem counterintuitive but, indeed, since the objective is to minimize the maximum workload, which is due to the critical tours of the more occupied caregiver, it is reasonable that the solver tends to schedule the uncertain requests so that a small number of them will be selected via the critical tours. And more flexibility we allow at the scheduling decision level, more possibility has the solver to include a small number of uncertain requests into the critical tours. Finally, Figure 12 shows that fixing the scheduling decisions determines a negligible deterioration in the committed approximation error. Therefore, the suggested decomposition seems to be a solid tool to gain in efficiency, still returning robust solutions of good quality, and so very promising to address robust Home Care instances of larger size. 


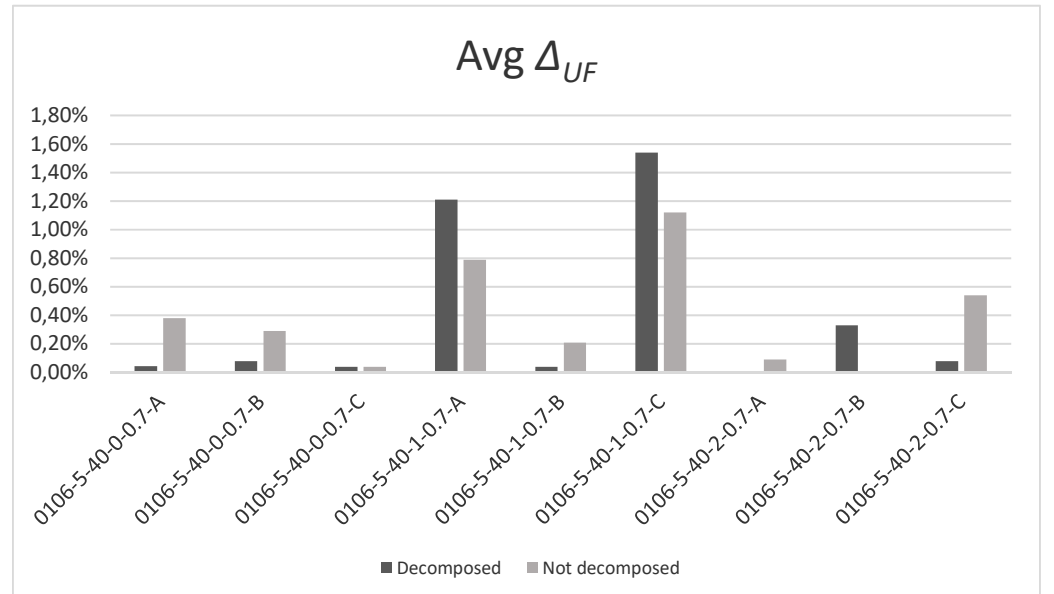

Figure 10: Decomposed vs not decomposed model - metric $\operatorname{Avg} \Delta_{U F}$

\section{Conclusions}

We studied the Home Care problem where caregiver-to-patient assignment, scheduling of patient requests, and caregiver routing are taken into account jointly over a given time horizon, and patient demand is subject to uncertainty. The study has been both methodologically and computationally oriented. In fact, we proposed a non-standard cardinality-constrained robust approach, which however is characterized by a difficult inner problem. Thus, an upper approximation is studied by stating and analysing some relevant properties induced by the suggested upper approximation. Then, we performed a wide computational experimentation, showing that the proposed approach is able to efficiently determine solutions of good quality, with a significant degree of robustness with respect to possible realizations of uncertain requests. We also proposed a decomposition approach that seems to be promising to face with even larger robust Home Care instances.

We plan to deeply study decomposition methods based on the robust approach proposed in this paper, and to experiment them on more, and larger, robust instances. We also plan to study alternative upper approximations of the cardinalityconstrained approach, both in theory and practice.

\section{Acknowledgments}

We thank the three anonymous referees and the Associate Editor for their interesting comments and suggestions which allowed us to greatly improve the first version of 


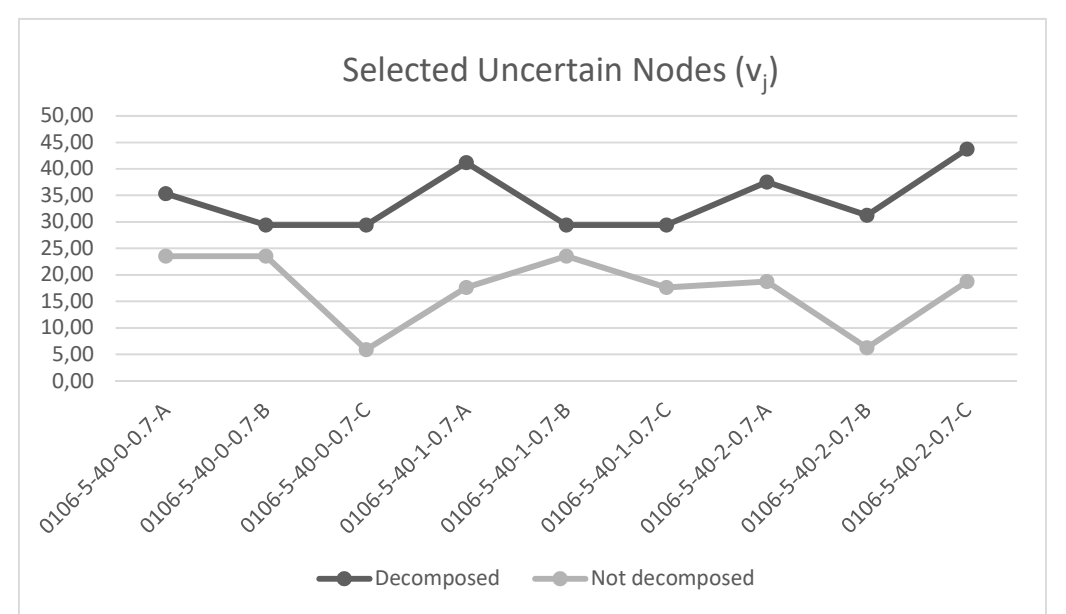

Figure 11: Decomposed vs not decomposed model - number of uncertain nodes selected in robust solutions

the paper.

\section{References}

[1] Bent, R.W., Van Hentenryck, P.: "Scenario-based planning for partially dynamic vehicle routing with stochastic customers", Operations Research, 52(6), 977-987 (2004)

[2] Bertels, S. and T. Fahle, "A hybrid setup for a hybrid scenario: combining heuristics for the home health care problem", Computers $\& 3$ Operations Research, 33, 2866-2890, 2006.

[3] Bertsimas, D., Sim, M.: The Price of Robustness. Operations Research. 52, $35-53(2004)$

[4] Binart, Dejax, Gendreau and Semet, "A 2-stage method for a field service routing problem with stochastic travel and service times", Computers $\mathcal{E}$ Operations Research, 65, 64-75, 2016.

[5] Bredström, D. and M. Rönnqvistr, "Combined vehicle routing and scheduling with temporal precedence and synchronization constraints", Eur. J. Oper. Res., 191, 19-31, 2008.

[6] Caceres-Cruz, J., Arias, P., Guimarans, D., Riera, D., Juan, A.A.: "Rich Vehicle Routing Problem: Survey", ACM Computing Surveys, 47(2), article number 32, (2015) 


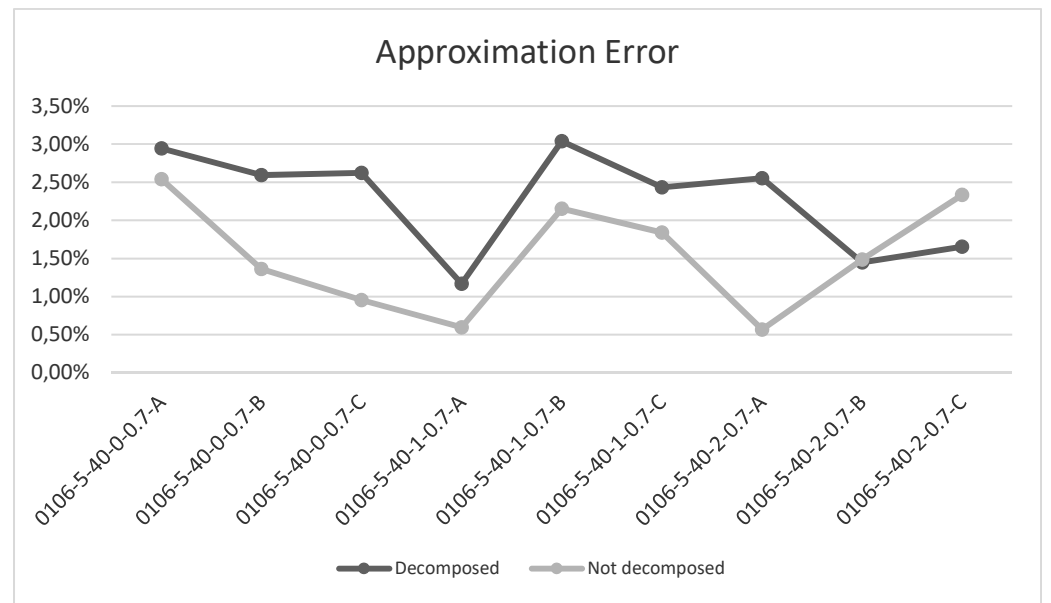

Figure 12: Decomposed vs not decomposed model - approximation error

[7] Cappanera, P., Scutellà, M.G.: "Joint assignment, scheduling, and routing models to home care optimization: A pattern-based approach", Transportation Science, 49(4), 830-852 (2015)

[8] Cappanera, P., Scutellà, M.G.: "Pattern generation policies to cope with robustness in Home Care", Springer Proceedings in Mathematics 83 Statistics, Proceedings of the International Conference on Health Care Systems Engineering, to appear.

[9] Cappanera, P., Scutellà, M.G., Visintin, F.: "Home Care Services delivery: equity versus efficiency in optimization models", Springer Proceedings in Mathematics $\&$ Statistics, 61, 1-15, Proceedings of the International Conference on Health Care Systems Engineering, Matta, Li, Sahin, Lanzarone and Fowler (Eds.), 2014.

[10] Carello, G., Lanzarone, E.: A cardinality-constrained robust model for the assignment problem in Home Care services. European Journal of Operational Research. 236, 748-762 (2014).

[11] Elbenani, B., Ferland, J.A. and V. Gascon, "Mathematical programming approach for routing home care nurses", Internat. Conf. Indust. Engrg. Management (IEEE, Washington, DC), 107-111, 2008.

[12] Erera, A.L., Morales, J.C., Savelsbergh, M.W.P.: "The vehicle routing problem with stochastic demand and duration constraints", Transportation Science, 44(4) 474-492 (2010)

[13] Eveborn, P., Flisberg, P. and M. Rönnqvist, "Laps care - and operational system for staff planning of home care", European Journal of Operational Research, 
171(3), 962-976, 2006.

[14] C. Fikar, \& P. Hirsch, "A matheuristic for routing real-world home service transport systems facilitating walking", Journal of Cleaner Production, 105, 300-310, 2015.

[15] C. Fikar, \& P. Hirsch, "Home health care routing and scheduling: A review.', Computers $\& 3$ Operations Research, 77, 86-95, 2017.

[16] Gamst, M., Jensen, T.S.: A branch-and-price algorithm for the long-term home care scheduling problem. Diethard Klatte, Hans-Jakob Lthi, Karl Schmedders, eds., Operations Research Proceedings 2011. Springer Berlin Heidelberg, 483-488 (2012)

[17] Groër, C., Golden, B., Wasil, E.: "The Consistent Vehicle Routing Problem", Manufacturing \&3 Service Operations Management, 11(4), 630-643 (2009)

[18] Hertz, A., Lahrichi, N.: A patient assignment algorithm for home care services. Journal of the Operational Research Society. 60(4), 481-495 (2009)

[19] M. Holte, \& C. Mannino, "The implementor/adversary algorithm for the cyclic and robust scheduling problem in health-care", European Journal of Operational Research 226, 551-559 (2013)

[20] E. Lanzarone, A. Matta, G. Scaccabarozzi, "A patient stochastic model to support human resource planning in home care", Production Planning and Control $21,3-25,2010$.

[21] Laporte, G., Louveaux, F. V., and Van Hamme, L., "An integer L-shaped algorithm for the capacitated vehicle routing problem with stochastic demands", Operations Research, 50(3), 415-423, 2002.

[22] E.L. Lawler, J.K. Lenstra, A.H.G. Rinnooy Kan, D.B. Shmoys, "The Travelling Salesman Problem", Wiley, Chichester (1985)

[23] R. Li, "Vehicle routing models in public safety and health care", Doctoral thesis, Information Systems, Statistics and Management Science, University of Alabama, USA, 2012.

[24] Liu, R., Xie, X., Garaix, T.: Hybridization of tabu search with feasible and infeasible local searches for periodic home health care logistics. Omega.47, 17-32 (2014)

[25] D. Mankowska, F. Meisel, \& C. Bierwirth, "The home health care routing and scheduling problem with interdependent services", Health Care Management Science 17(1), 15-30 (2014)

[26] T.V.L. Nguyen, N.E. Toklu, \& R. Montemanni, "Matheuristic optimization for robust home health care services", Lecture Notes in Management Science 7, 1-7 (2015) 
[27] Nickel, S., Schröder, M., Steeg, J.: Mid-term and short-term planning support for home health care services. European Journal of Operational Research. 219(3) $574-587$ (2012)

[28] Rasmussen, M.S., Justesen, T., Dohn, A. and J. Larsen, "The Home Care Crew Scheduling Problem: Preference-based visit clustering and temporal dependencies", European Journal of Operational Research 219, 598-610 (2012)

[29] K.D. Rest, A. Trautsamwieser, \& P. Hirsch, "Trends and risk in home health care", Journal of Humanitarian Logist Supply Chain Manage 2, 34-53 (2012)

[30] C. Rodriguez, T. Garaix, X., Xie \& V. Augusto, "Staff dimensioning in homecare services with uncertain demands", Int J Prod, 53(24), 7396-7410 (2015)

[31] S. Souyris, C. E. Cortés, F. Ordóñez \& A. Weintraub, "A robust optimization approach to dispatching technicians under stochastic service times", Optimization Letters 7, 1549-1568 (2013)

[32] Trautsamwieser, A., Hirsch, P.: A branch-price-and-cut approach for solving the medium-term home health care planning problem. Networks. 64(3), 143-159 (2014)

[33] L. A. Wolsey, Integer programming, Wiley-Interscience Series in Discrete Mathematics and Optimization, John Wiley \& Sons, New York (1998)

[34] Yalçındağ, S., Cappanera, P., Scutellà, M.G., Matta, A., Şahin, E.: PatternBased Decompositions for Human Resource Planning in Home Health Care Services. Computers \& Operations Research. 73, 12-26 (2016)

[35] Yuan, B., Liu, R., Jiang, Z.: Home health care crew scheduling and routing problem with stochastic service times. Proceedings of 2014 IEEE International Conference on Automation Science and Engineering. 564-560 (2014) 


\section{Appendix A. The linearized model}

Denote with $\zeta^{\omega d}$ the dual variable associated with (14), and with $\pi_{j}^{\omega d}$ the dual variables associated with $v_{j}^{\omega d} \leq 1$ in the linear relaxation of $(13)-(15)$. Then the feasible solutions to the $s R H C_{\Gamma}$ approximation can be modelled as follows:

$$
\begin{aligned}
& \sum_{(i, j) \in A} \sum_{\omega \in O_{d}} x_{i j}^{\omega d} \leq \sum_{p \in P_{j}: p(d) \geq 1} z_{j p} \\
& \forall j \in N, \forall d \in W \\
& \sum \sum_{(i, j) \in A} x_{i j}^{\omega d} \geq \sum z_{j p}: z \quad \forall j \in N, \forall d \in W, \forall k \in K \\
& \sum_{p \in P_{j}} z_{j p}=1 \\
& \forall j \in N \\
& \sum_{\omega \in O} u_{\omega j} \leq T \\
& \forall j \in N \\
& x_{i j}^{\omega d} \leq u_{\omega j} \\
& \forall(i, j) \in A, \forall j \in N, \forall d \in W, \forall \omega \in O_{d} \\
& u_{\omega j} \leq \sum_{(i, j) \in A} \sum_{d \in W} x_{i j}^{\omega d} \\
& j \in N, \forall \omega \in O \\
& \bar{R}_{S \Gamma}^{\omega d}=\sum_{\substack{(i, j) \in A: \\
i, j \in \bar{N}^{+}}}\left(t_{i j}+a_{j}\right) \cdot x_{i j}^{\omega d}+\sum_{\substack{(i, j) \in A: \\
i \in \bar{N}, j \in \bar{N}^{+}}}\left(t_{j}^{\max }+a_{j}\right) \cdot x_{i j}^{\omega d}+ \\
& \Gamma \cdot \zeta^{w d}+\sum_{j \in \tilde{N}} \pi_{j}^{\omega d} \leq D_{\omega} \\
& \sum_{(i, j) \in A} x_{i j}^{\omega d}=\sum_{(j, i) \in A} x_{j i}^{\omega d} \\
& \sum_{(0, j) \in A} y_{0 j}^{d}=\sum_{j \in N} \sum_{p \in P_{j}: p(d) \geq 1} z_{j p} \\
& \sum_{(i, j) \in A} y_{i j}^{d}-\sum_{(j, i) \in A} y_{j i}^{d}=\sum_{p \in P_{j}: p(d) \geq 1} z_{j p} \\
& y_{i j}^{d} \leq 2 n \sum_{\omega \in O_{d}} x_{i j}^{\omega d} \\
& \pi_{j}^{\omega d}+\zeta^{\omega d} \geq \sum_{\substack{(i, j) \in A: \\
i \in \bar{N}^{+}}}\left(t_{i j}+a_{j}\right) \cdot x_{i j}^{\omega d}+ \\
& \sum_{\substack{(j, i) \in A: \\
i \in \bar{N}^{+}}}\left(t_{j i}-t_{i}^{\max }\right) \cdot x_{j i}^{\omega d}+\sum_{\substack{(i, j) \in A: \\
i \in \tilde{N}}}\left(t_{j}^{\max }+a_{j}\right) \cdot x_{i j}^{\omega d} \quad \forall d \in W, \forall \omega \in O_{d}, \forall j \in \tilde{N} \\
& \pi_{j}^{\omega d} \geq 0 \\
& \zeta^{\omega d} \geq 0 \\
& \forall j \in \tilde{N}, \forall d \in W, \forall \omega \in O_{d} \\
& \forall d \in W, \forall \omega \in O_{d} .
\end{aligned}
$$




\section{Appendix B. Maximizing the minimum caregiver utilization fac- tor}

We formally show that the balancing objective function maxmin, which maximizes the minimum caregiver utilization factor, cannot be used in the same form investigated in [7] and [34], since the corresponding optimal value is always 1 (constraint numbers refer to Appendix A).

Proposition 4 The optimal value of (20)-(33) under the objective function maxmin, i.e.:

$$
\begin{aligned}
& \max m \\
& \text { (20)-(33) } \\
& \sum_{d \in W} \bar{R}_{S \Gamma}^{\omega d} \\
& \frac{d \in W}{|W| \cdot D_{\omega}} \geq m, \quad \forall \omega \in O,
\end{aligned}
$$

is always 1.

Proof: Consider the standard technique used in Robust Optimization, i.e. the one for which each inner ILP model (13) - (15) is replaced by its Linear Programming relaxation and so, by strong duality, by its LP dual. Such a technique fails when addressing the maxmin objective function. In fact, in order to maximize $m$, the solver assigns to the left-hand-side of each constraint (26) the maximum possible value, i.e. $D_{\omega}$ (instead of assigning the minimum possible value, i.e. $\bar{R}_{S \Gamma}^{\omega d}$, as stated in (34)) and therefore the optimal objective function value is always 1 .

\section{Appendix C. Estimating the matheuristic approximation error}

We provide a proof of Proposition 3.

Proof: Let $C^{*}$ be a complete set of tours representing a feasible solution to $s R H C_{\Gamma}$, and consider the critical tours corresponding to the complete tours in $C^{*}$. Without loss of generality assume that, for each such critical tour, the certain nodes precede the selected uncertain nodes within each cluster. Remember in fact that, due to the distance structure, any permutation of a subset of patients in the same cluster induces the same identical workload contribution to the objective function.

Now, let us evaluate the objective function value of $C^{*}$ according to the proposed upper approximation. That is, by computing $\bar{R}_{S \Gamma}^{\omega d}$ for the critical tour of each operator $\omega$ on each day $d$. This is obtained by replacing, in the formula expressing the exact workload of the tour, both the exact travelling time of each arc 
$(i, j)$ linking two selected uncertain nodes, and the exact travelling time of each arc $(i, j)$ not belonging to the corresponding complete tour, by the over-estimate $t_{j}^{\max }$. It is immediate to observe that the first group is composed by at most $(\Gamma-1)$ arcs, since the total number of selected uncertain nodes is $\Gamma$. On the other hand, the second group is composed by at most $N_{c}$ arcs, since these are arcs linking the different clusters and, due to the inequalities (18), for each cluster, and in each day of the planning horizon, each available operator enters each cluster at most once. Therefore, for each critical tour, the absolute error is bounded from above by $N_{c}\left(T_{c}^{\max }-T_{c}^{\min }\right)+(\Gamma-1)\left(T_{c}^{\max }-\delta\right)$. The thesis follows by considering the overall set of caregivers over the addressed time horizon.

Table 3 reports, for each instance group characterized by the same number of clusters and patients, the maximum estimated travel time (EstMaxTT) computed as described in Proposition 3 and the average integer-rounded travel time (AvgTT) over all the instances of the group returned by the robust model, as $\Gamma$ varies. Travel time refers to the total travel time spent by all of the caregivers in the whole planning horizon. The parameters required to compute the absolute error are given in columns 2-5. Observe that, with 5 clusters and $\Gamma=1$, the ratio between AvgTT and EstMaxTT is not greater than 0.52 independently of the number of patients considered, i.e. 40 or 60 . For 8 clusters and $\Gamma=1$, such a ratio is smaller and it does not exceed 0.34 . When $\Gamma=2$ is considered, these ratios tend to decrease; specifically, they are 0.42 for $N_{c}=5$ and 0.30 for $N_{c}=8$.

Table 3: Estimate given by Proposition 3

\begin{tabular}{lccccrrrr}
\cline { 4 - 8 } & & & \multicolumn{4}{c}{$\Gamma=1$} \\
\hline Instance groups & $T_{c}^{\text {min }}$ & $T_{c}^{\text {max }}$ & $\delta$ & $N_{c}$ & EstMaxTT & AvgTT & EstMaxTT & AvgTT \\
\hline $0106-5-40$ & 5 & 19 & 3 & 5 & 700 & 352 & 860 & 362 \\
$0106-8-40$ & 4 & 21 & 3 & 8 & 1360 & 395 & 1540 & 466 \\
$0106-5-60$ & 5 & 21 & 3 & 5 & 1200 & 633 & & \\
$0106-8-60$ & 4 & 21 & 3 & 8 & 2040 & 688 & & \\
\hline
\end{tabular}

